\title{
Dynamics of bright and dark multi-soliton solutions for two higher-order Toda lattice equations for nonlinear waves
}

\author{
Nan Liu', Xiao-Yong Wen ${ }^{1 *}$ and Ling $\mathrm{Xu}^{1}$
}

\section{"Correspondence:}

xiaoyongwen@163.com

'School of Applied Science, Beijing

Information Science and

Technology University, Beijing,

China

\begin{abstract}
Discrete $\mathrm{N}$-fold Darboux transformation (DT) is used to derive new bright and dark multi-soliton solutions of two higher-order Toda lattice equations. Propagation and elastic interaction structures of such soliton solutions are shown graphically. The details of their evolutions are studied via numerical simulations. Numerical results show the accuracy of our numerical scheme and the stable evolutions of such bright and dark multi-solitons without a noise. To compare the numerical evolution results with the classical Toda lattice equation, we also investigate the dynamical behaviors of the multi-soliton solutions for Toda lattice equation via numerical simulations, and we find that the multi-soliton solutions of Toda lattice equation have better stability and are more robust against a big noise than its two corresponding higher-order equations. The same small noise has different effect on the evolutions of the multi-soliton solutions for three different equations in the same hierarchy. The possible reason is that the higher-order nonlinear terms of the higher-order equation cause the instability of the wave propagation. The discrete generalized $(n, N-n)$-fold DTs are constructed and used to derive some discrete rational solutions of three equations, and a few mathematical features for such rational solutions are also discussed. Results might be helpful for understanding the propagation of nonlinear waves in soliton theory.
\end{abstract}

Keywords: Higher-order Toda lattice equation; Discrete N-fold Darboux transformation; Discrete bright and dark multi-soliton solutions; Rational solutions; Numerical simulations

\section{Introduction}

Recently, the nonlinear lattice equations (NLEs) [1-4], treated as the spatially discrete analogues of the nonlinear partial differential equations (NPDEs) [5-9], have received certain attention [10-14]. Dynamical behaviors of solitons in the continuous and discrete cases are described by the NPDEs and NLEs [10], respectively. The NLEs [15-17] can be taken as the models of some physical phenomena and may be used to describe many physical situations. For example, the Toda lattice equation is a simple model for a one-dimensional crystal in solid state physics $[11,12]$; the self-dual network equations describe the propagation of electrical signals in a cascade of four-terminal nonlinear LC self-dual circuits or the wave propagation in a nonlinear, lumped, self-dual ladder type network [13]; the Ablowitz-Ladik lattice equation can describe the interaction and propagation of optical

(c) The Author(s) 2018. This article is distributed under the terms of the Creative Commons Attribution 4.0 International License (http://creativecommons.org/licenses/by/4.0/), which permits unrestricted use, distribution, and reproduction in any medium, provided you give appropriate credit to the original author(s) and the source, provide a link to the Creative Commons license, and indicate if changes were made. 
pulses in a nonlinear waveguide array [14]; the Volterra lattice system is in connection with the spectrum of Langmuir wave in plasma dynamics [10]. Searching for explicit exact solutions of NLEs is a very significant work. Exact solutions can make people understand deeply the physical mechanism of the natural phenomena described by NLEs. Some methods for constructing explicit exact solutions of NLEs, such as the inverse scattering method [1-4, 18, 19], Hirota method [13, 20], Bäcklund transformation [21-23], and Darboux transformation (DT) [24-41], have been developed. Among them, the DT based on the Lax pair is an algebraic method which is an effective tool to solve the Lax integrable NLEs. The main idea of the DT method is to keep the linear eigenvalue problems of the integrable NLEs invariant. In fact, the Darboux transformation exhibits different forms such as a general dressing procedure (see, e.g., Ref. [26]), the iterated DT with the iteration operator form (see, e.g., Ref. [24, 25]), the iterated DT with the iterated Darboux matrix (see, e.g., Refs. [27-30]), the DT with the first-order Darboux matrix (see, e.g., Refs. [31-33]), and the DT with the $N$-order Darboux matrix whose elements are the polynomials of the spectral parameters (see, e.g., Refs. [34-41]), etc. Theoretically, the solution obtained by a single DT (i.e., 1 -fold DT which is the $N$-fold DT when $N=1$ ) can be taken as the new starting point from which to derive another solution by making a DT again, hence a series of explicit solutions can be generated by iteration step by step [35-37]. However, it is very difficult to carry out the iterative process. Compared with the iterated DT, the $N$-fold DT technique with the $N$-order Darboux matrix is an effective tool to solve the Lax integrable NLEs and its main advantage is to give multi-soliton solutions without complicated iterative process [35-39].

For the NLEs, Toda lattice equation is one of the well-known Lax integrable NLEs which well describes the soliton physical phenomena such as nonlinear wave propagation and nonergodic phenomena in Fermi-Pasta-Ulam recurrence [11, 12]. The main aim of the present paper is a detailed study of bright and dark multi-soliton solutions and their dynamical behaviors of two higher-order NLEs associated with the following Toda lattice equation [42]:

$$
\left\{\begin{array}{l}
u_{n, t}=v_{n+1}-v_{n}, \\
v_{n, t}=v_{n}\left(u_{n}-u_{n-1}\right),
\end{array}\right.
$$

where $u_{n}=u(n, t), v_{n}=v_{(n, t)}$ are the functions of a discrete variable $n$ and the time variable $t$ and $u_{n, t}=\frac{d u_{n}}{d t}, v_{n, t}=\frac{d v_{n}}{d t}$. Equation (1) is an important discrete Lax integrable NLE and it is connected with the following discrete linear spectral problem [42]:

$$
\begin{array}{ll}
E \psi_{n}=M_{n} \psi_{n}, & M_{n}=\left(\begin{array}{ll}
0 & 0 \\
0 & 1
\end{array}\right) \lambda+\left(\begin{array}{cc}
0 & 1 \\
-v_{n} & -u_{n}
\end{array}\right), \\
\psi_{n, t}=U_{n} \psi_{n}, & U_{n}=\left(\begin{array}{cc}
-1 & 0 \\
0 & 0
\end{array}\right) \lambda+\left(\begin{array}{cc}
u_{n-1}+1 & 1 \\
-v_{n} & 1
\end{array}\right) .
\end{array}
$$

Equation (1) can be taken as the discrete approximation of the Korteweg-de Vries (KdV) equation in fluids and also has served as a useful guide in the studies on nonlinear waves $[10,43]$. Under the transformations $u_{n}=-Q_{n, t}, v_{n}=e^{Q_{n-1}-Q_{n}}$, Eq. (1) can be transformed 
to our familiar classic form

$$
Q_{n, t t}=e^{Q_{n-1}-Q_{n}}-e^{Q_{n}-Q_{n+1}},
$$

where $Q_{n}=Q(n, t)$ is the displacement of the $n$th particle from its equilibrium position [12]. To understand many nonlinear, nonergodic phenomena such as Fermi-Pasta-Ulam recurrence, Toda proposed a one-dimensional nonlinear lattice model with neighboring particles interacting through an exponential potential function, and the single soliton solution and the two-soliton solution have been obtained [12, 44]. In Ref. [19], the general $\mathrm{N}$-soliton formula is derived by use of an inverse-scattering method, and constants of the motion are expressed in terms of the scattering data. In Ref. [21], Bäcklund transformation, superposition formula, and multi-soliton solutions have been constructed for Eq. (4) with some discussion of its generalizations. In Refs. [33, 35], one-soliton solution and the even-order soliton solutions for Eq. (1) have been obtained via a single DT and $N$-fold DT, respectively. In Ref. [45], the existence of motion integrals is proved by a different method which shows the Toda lattice equation to be a finite-dimensional analog of the Korteweg-de Vries partial differential equation. In Ref. [46], some new kinds of solitonlike solutions have been given by a detailed study of limits of $N$-soliton solutions of the Toda lattice as $N$ tends to infinity. In Ref. [47], an explicit solution formula in terms of determinants for Eq. (4) has been derived with operator methods. In Ref. [48], some new generalized soliton solutions for the Toda lattice equations based on the invariance of Gibbon and Tabor's equation under the fractional linear transformation have been given. In Ref. [49], some quasi-periodic and periodic solutions in terms of hyperelliptic $\sigma$ functions for arbitrary genus have been given by Toda's original approach. In Ref. [50], the soliton solution in the Wronskian form for Eq. (4) has been obtained. In Ref. [51], some approximate soliton solutions around an exact soliton solution of Eq. (4) have been given. In Refs. [52, 53], some complexiton solutions and rational solutions in Casoratian form of Eq. (4) have been given. In Ref. [54], a kind of explicit quasi-periodic solution and its limit have been derived. In Ref. [42], a Toda lattice hierarchy has been put forward from a discrete matrix spectral problem, in which Eq. (1) is the first member of this hierarchy, while the second and third equations in this hierarchy are listed as follows [42]:

$$
\begin{aligned}
& \left\{\begin{aligned}
u_{n, t}= & v_{n+1}\left(u_{n}+u_{n+1}\right)-v_{n}\left(u_{n}+u_{n-1}\right), \\
v_{n, t}= & v_{n}\left(u_{n}^{2}-u_{n-1}^{2}-v_{n-1}+v_{n+1}\right),
\end{aligned}\right. \\
& \left\{\begin{aligned}
u_{n, t}= & v_{n}\left(v_{n-1}+v_{n}+u_{n-1}^{2}+u_{n} u_{n-1}+u_{n}^{2}\right) \\
& -v_{n+1}\left(v_{n+1}+v_{n+2}+u_{n+1}^{2}+u_{n} u_{n+1}+u_{n}^{2}\right), \\
v_{n, t}= & v_{n}\left(u_{n-1}^{3}+u_{n-1} v_{n}+2 u_{n-1} v_{n-1}+u_{n-2} v_{n-1}\right. \\
& \left.-u_{n+1} v_{n+1}-2 u_{n} v_{n+1}-u_{n} v_{n}-u_{n}^{3}\right),
\end{aligned}\right.
\end{aligned}
$$

where $u_{n}=u(n, t)$ and $v_{n}=v(n, t)$ are the functions of the discrete variable $n$ and time variable $t, u_{n, t}=\frac{d u_{n}}{d t}, v_{n, t}=\frac{d v_{n}}{d t}$. Here, we call Eqs. (5) and (6) the higher-order Toda lattice equations relative to Toda lattice equation. Just like the higher-order equations of $\mathrm{KdV}$ hierarchy can also describe the motion of nonlinear shallow water waves [55], we believe that Eqs. (5) and (6) may be used to describe some physical phenomena for nonlinear 
waves because they belong to the same hierarchy of Toda lattice equation. According to Refs. [42, 43], Eqs. (5) and (6) have the same space part of the discrete linear spectral problem (i.e., Lax pair) as Eq. (1), and their corresponding time parts are listed as follows:

$$
\begin{aligned}
\psi_{n, t}= & W_{n} \psi_{n}, \\
W_{n}= & \left(\begin{array}{cc}
-1 & 0 \\
0 & 0
\end{array}\right) \lambda^{2}+\left(\begin{array}{cc}
1 & 1 \\
-v_{n} \lambda & 1
\end{array}\right) \lambda+\left(\begin{array}{cc}
u_{n-1}^{2}+v_{n-1} & u_{n-1} \\
-u_{n} v_{n} & v_{n}
\end{array}\right), \\
\psi_{n, t}= & V_{n} \psi_{n}, \\
V_{n}= & \left(\begin{array}{cc}
\frac{1}{2} & 0 \\
0 & -\frac{1}{2}
\end{array}\right) \lambda^{3}+\left(\begin{array}{cc}
0 & -1 \\
v_{n} & 0
\end{array}\right) \lambda^{2}+\left(\begin{array}{cc}
v_{n} & -u_{n-1} \\
u_{n} v_{n} & -v_{n}
\end{array}\right) \lambda \\
& +\left(\begin{array}{rr}
-u_{n-1} v_{n}-2 u_{n-1} v_{n-1}-u_{n-2} v_{n-1}-u_{n-1}^{3} & -v_{n-1}-v_{n}-u_{n-1}^{2} \\
v_{n}^{2}+v_{n} v_{n+1}+u_{n}^{2} v_{n} & -u_{n-1} v_{n}-u_{n} v_{n}
\end{array}\right) .
\end{aligned}
$$

The compatibility conditions between (2) and (7) (i.e., $M_{n, t}+M_{n} W_{n}-W_{n+1} M_{n}=0$ ) and between (2) and (8) (i.e., $M_{n, t}+M_{n} V_{n}-V_{n+1} M_{n}=0$ ) lead to Eqs. (5) and (6), respectively. In Ref. [42], the Hamiltonian structures of the hierarchy associated with Eqs. (1), (5), and (6) has been given by a trace identity technique. In Ref. [43], the concrete infinitely many conservation laws of Eqs. (1) and (5) have been constructed based on their linear spectral problems. However, as we know, bright and dark multi-soliton solutions, relevant elastic interactions, and dynamical behaviors for Eqs. (5) and (6) and rational solutions in terms of determinants for Eqs. (1), (5), and (6) have not been investigated previously.

Different from the previous studies, in this paper, we further investigate Eqs. (5) and (6) via the discrete $N$-fold DT technique. The rest of this paper is arranged as follows: In Sect. 2, a discrete version of the $N$-fold DT for Eqs. (5) and (6) is constructed based on their Lax pair. In Sect. 3, discrete bright and dark multi-soliton solutions in terms of determinant of Eq. (5) are derived via the resulting $N$-fold DT from non-vanishing background, and the solitonic elastic interactions are analyzed graphically, the dynamical behaviors of such soliton solutions are investigated via numerical simulations. In Sect. 4, the dynamical behaviors of discrete multi-soliton solutions of Eq. (6) are investigated via numerical simulations. In Sect. 5, the dynamical behaviors of multi-soliton solutions of Eq. (1) are investigated via numerical simulations. In Sect. 6, based on the $N$-fold DT in Sect. 2, we propose a discrete version of the generalized $(n, N-n)$-fold DT for Eqs. (1), (5), and (6). In Sect. 7, some discrete rational solutions in terms of determinants of the first equation are given by means of the generalized $(1, N-1)$-fold DT, and a few mathematical features are discussed for such rational solutions. Finally, we conclude this paper in the final section.

\section{Discrete $\mathbf{N}$-fold Darboux transformation}

In this section, we will construct the discrete $N$-fold DT of Eqs. (5) and (6). We introduce the following gauge transformation:

$$
\widetilde{\psi}_{n}=T_{n} \psi_{n}
$$


where $\widetilde{\psi}_{n}$ is required to satisfy Eqs. (2), (7), and (8) with $M_{n}, W_{n}, V_{n}$ replaced by $\widetilde{M}_{n}, \widetilde{W}_{n}$, $\widetilde{V}_{n}$, i.e.,

$$
\begin{array}{ll}
E \widetilde{\psi}_{n}=\widetilde{M}_{n} \widetilde{\psi}_{n}, & \widetilde{M}_{n}=T_{n+1} M_{n} T_{n}^{-1}, \\
\widetilde{\psi}_{n, t}=\widetilde{W}_{n} \widetilde{\psi}_{n}, & \widetilde{W}_{n}=\left(T_{n, t}+T_{n} W_{n}\right) T_{n}^{-1}, \\
\widetilde{\psi}_{n, t}=\widetilde{V}_{n} \widetilde{\psi}_{n}, & \widetilde{V}_{n}=\left(T_{n, t}+T_{n} V_{n}\right) T_{n}^{-1} .
\end{array}
$$

To construct the $N$-fold DT, $\widetilde{M}_{n}, \widetilde{W}_{n}$, and $\widetilde{V}_{n}$ must have the same forms as $M_{n}, W_{n}$, and $V_{n}$, respectively, except replacing the old potentials $u_{n}, v_{n}$ with the new ones $\widetilde{u}_{n}, \widetilde{v}_{n}$. So, proper $T_{n}$ will ensure the correctness of the $N$-fold DT. Hereby, a special $N$-order Darboux matrix $T_{n}$ is defined as

$$
T_{n}=\left(\begin{array}{ll}
a_{n} & b_{n} \\
c_{n} & d_{n}
\end{array}\right)=\left(\begin{array}{cc}
\lambda^{N}\left(1-b_{n}^{(N-1)}\right)+\sum_{j=0}^{N-1} a_{n}^{(j)} \lambda^{j} & \sum_{j=0}^{N-1} b_{n}^{(j)} \lambda^{j} \\
\sum_{j=0}^{N-1} c_{n}^{(j)} \lambda^{j} & \lambda^{N}+\sum_{j=0}^{N-1} d_{n}^{(j)} \lambda^{j}
\end{array}\right),
$$

in which $N$ is a positive integer, $a_{n}^{(j)}, b_{n}^{(j)}, c_{n}^{(j)}$, and $d_{n}^{(j)}$, which are the functions of the variables $n$ and $t$, can be determined by the following linear algebraic system:

$$
\left\{\begin{array}{l}
-b_{n}^{(N-1)} \lambda_{i}^{N}+\sum_{j=0}^{N-1} a_{n}^{(j)} \lambda_{i}^{j}+\sum_{j=0}^{N-1} b_{n}^{(j)} \delta_{i, n} \lambda_{i}^{j}=-\lambda_{i}^{N}, \\
\sum_{j=0}^{N-1} c_{n}^{(j)} \lambda_{i}^{j}+\sum_{j=0}^{N-1} d_{n}^{(j)} \delta_{i, n} \lambda_{i}^{j}=-\lambda_{i}^{N} \delta_{i, n}
\end{array}\right.
$$

where

$$
\delta_{i, n}=\frac{\varphi_{2, n}\left(\lambda_{i}\right)-r_{i} \psi_{2, n}\left(\lambda_{i}\right)}{\varphi_{1, n}\left(\lambda_{i}\right)-r_{i} \psi_{1, n}\left(\lambda_{i}\right)}, \quad 1 \leq i \leq 2 N
$$

in which $\varphi_{n}\left(\lambda_{i}\right)=\left(\varphi_{1, n}\left(\lambda_{i}\right), \varphi_{2, n}\left(\lambda_{i}\right)\right)^{T}$ and $\psi_{n}\left(\lambda_{i}\right)=\left(\psi_{1, n}\left(\lambda_{i}\right), \psi_{2, n}\left(\lambda_{i}\right)\right)^{T}$ are two essential solutions of (2), (3), (7), and (8), where $\lambda=\lambda_{i}(i=1,2, \ldots, 2 N), r_{i}(i=1,2, \ldots, 2 N)$ are $2 N$ arbitrary real constants. When we choose the $2 N$ different real parameters $\lambda_{i}\left(\lambda_{i} \neq \lambda_{j}, i \neq j\right)$ so that the determinant of the coefficients for (14) is nonzero, the matrix $T_{n}$ is determined by (14) uniquely.

Equation (13) makes clear that $\operatorname{det} T_{n}$ is the (2N)th order polynomial of $\lambda$ and

$$
\operatorname{det} T_{n}\left(\lambda_{i}\right)=a_{n}\left(\lambda_{i}\right) d_{n}\left(\lambda_{i}\right)-b_{n}\left(\lambda_{i}\right) c_{n}\left(\lambda_{i}\right)
$$

Moreover, from (9), (13), and (14), it follows that

$$
a_{n}\left(\lambda_{i}\right)=-b_{n}\left(\lambda_{i}\right) \delta_{i, n}, \quad c_{n}\left(\lambda_{i}\right)=-d_{n}\left(\lambda_{i}\right) \delta_{i, n} .
$$

Hence

$$
\operatorname{det} T_{n}\left(\lambda_{i}\right)=0 .
$$

In other words, $\lambda_{i}\left(\lambda_{i} \neq 0\right)(i=1,2, \ldots, 2 N)$ are the $2 N$ roots of the $\operatorname{det} T_{n}$, namely

$$
\operatorname{det} T_{n}=\left(1-b_{n}^{(N-1)}\right) \prod_{i=1}^{2 N}\left(\lambda-\lambda_{i}\right) .
$$

By using the above facts, we have the following theorem. 
Theorem 1 If the old potential $\left(u_{n}, v_{n}\right)$ is a solution of Eqs. (5) and (6), then the new potential $\left(\widetilde{u}_{n}, \widetilde{v}_{n}\right)$ with

$$
\widetilde{u}_{n}=u_{n}+d_{n}^{(N-1)}-d_{n+1}^{(N-1)}, \quad \widetilde{v}_{n}=\frac{v_{n}+c_{n}^{(N-1)}}{1-b_{n}^{(N-1)}}
$$

is also a solution of Eqs. (5) and (6), in which

$$
b_{n}^{(N-1)}=\frac{\Delta b_{n}^{(N-1)}}{\Delta_{1}}, \quad c_{n}^{(N-1)}=\frac{\Delta c_{n}^{(N-1)}}{\Delta_{2}}, \quad d_{n}^{(N-1)}=\frac{\Delta d_{n}^{(N-1)}}{\Delta_{2}},
$$

where

$$
\begin{aligned}
\Delta_{1} & =\left|\begin{array}{cccccccc}
1 & \lambda_{1} & \cdots & \lambda_{1}^{N-1} & \delta_{1, n} & \lambda_{1} \delta_{1, n} & \cdots & \lambda_{1}^{N-1} \delta_{1, n}-\lambda_{1}^{N} \\
1 & \lambda_{2} & \cdots & \lambda_{2}^{N-1} & \delta_{2, n} & \lambda_{2} \delta_{2, n} & \cdots & \lambda_{2}^{N-1} \delta_{2, n}-\lambda_{2}^{N} \\
\cdots & \cdots & \cdots & \cdots & \cdots & \cdots & \cdots & \cdots \\
1 & \lambda_{2 N} & \cdots & \lambda_{2 N}^{N-1} & \delta_{2 N, n} & \lambda_{2 N} \delta_{2 N, n} & \cdots & \lambda_{2 N}^{N-1} \delta_{2 N, n}-\lambda_{2 N}^{N}
\end{array}\right|, \\
\Delta_{2} & =\left|\begin{array}{cccccccc}
1 & \lambda_{1} & \cdots & \lambda_{1}^{N-1} & \delta_{1, n} & \lambda_{1} \delta_{1, n} & \cdots & \lambda_{1}^{N-1} \delta_{1, n} \\
1 & \lambda_{2} & \cdots & \lambda_{2}^{N-1} & \delta_{2, n} & \lambda_{2} \delta_{2, n} & \cdots & \lambda_{2}^{N-1} \delta_{2, n} \\
\cdots & \cdots & \cdots & \cdots & \cdots & \cdots & \cdots & \cdots \\
1 & \lambda_{2 N} & \cdots & \lambda_{2 N}^{N-1} & \delta_{2 N, n} & \lambda_{2 N} \delta_{2 N, n} & \cdots & \lambda_{2 N}^{N-1} \delta_{2 N, n}
\end{array}\right|,
\end{aligned}
$$

and $\Delta b_{n}^{(N-1)}$ is produced from $\Delta_{1}$ by replacing its $(2 N)$ th column with $\left(-\lambda_{1}^{N},-\lambda_{2}^{N}, \ldots\right.$, $\left.-\lambda_{2 N}^{N}\right)^{T}, \Delta c_{n}^{(N-1)}$ and $\Delta d_{n}^{(N-1)}$ are produced from $\Delta_{2}$ by replacing their $N$ th and $(2 N)$ th columns with $\left(-\delta_{1, n} \lambda_{1}^{N},-\delta_{2, n} \lambda_{2}^{N}, \ldots,-\delta_{2 N, n} \lambda_{2 N}^{N}\right)^{T}$, respectively, while $\Delta d_{n+1}^{(N)}$ can be derived from $\Delta d_{n}^{(N)}$ by substituting $n+1$ for $n$. Thus, matrices $\widetilde{M}_{n}, \widetilde{W}_{n}$, and $\widetilde{V}_{n}$ in Eqs. (10), (11), and (12) have the same forms as $M_{n}, W_{n}$, and $V_{n}$, respectively, namely

$$
\begin{aligned}
\widetilde{M}_{n}= & \left(\begin{array}{cc}
0 & 1 \\
-\widetilde{v}_{n} & \lambda-\widetilde{u}_{n}
\end{array}\right), \quad \widetilde{W}_{n}=\left(\begin{array}{cc}
-\lambda^{2}+\lambda+\widetilde{v}_{n-1}+\widetilde{u}_{n-1}^{2} & \lambda+\widetilde{u}_{n-1} \\
-\widetilde{v}_{n}\left(\lambda+\widetilde{u}_{n}\right) & \lambda+\widetilde{v}_{n}
\end{array}\right), \\
\widetilde{V}_{n}= & \left(\begin{array}{cc}
\frac{1}{2} & 0 \\
0 & -\frac{1}{2}
\end{array}\right) \lambda^{3}+\left(\begin{array}{cc}
0 & -1 \\
\widetilde{v}_{n} & 0
\end{array}\right) \lambda^{2}+\left(\begin{array}{cc}
\widetilde{v}_{n} & -\widetilde{u}_{n-1} \\
\widetilde{u}_{n} \widetilde{v}_{n} & -\widetilde{v}_{n}
\end{array}\right) \lambda \\
& +\left(\begin{array}{cc}
-\widetilde{u}_{n-1} \widetilde{v}_{n}-2 \widetilde{u}_{n-1} \widetilde{v}_{n-1}-\widetilde{u}_{n-2} \widetilde{v}_{n-1}-\widetilde{u}_{n-1}^{3} & -\widetilde{v}_{n-1}-\widetilde{v}_{n}-\widetilde{u}_{n-1}^{2} \\
\widetilde{v}_{n}^{2}+\widetilde{v}_{n} \widetilde{v}_{n+1}+\widetilde{u}_{n}^{2} \widetilde{v}_{n} & -\widetilde{u}_{n-1} \widetilde{v}_{n}-\widetilde{u}_{n} \widetilde{v}_{n}
\end{array}\right) .
\end{aligned}
$$

Proof Let $T_{n}^{-1}=T_{n}^{*} / \operatorname{det} T_{n}$ and

$$
F(\lambda)=T_{n+1} M_{n} T_{n}{ }^{*}=\left(\begin{array}{ll}
f_{11}(\lambda, n) & f_{12}(\lambda, n) \\
f_{21}(\lambda, n) & f_{22}(\lambda, n)
\end{array}\right) .
$$

It can be verified that $f_{22}(\lambda, n)$ is the $(2 N+1)$ th order polynomial in $\lambda, f_{11}(\lambda, n), f_{12}(\lambda, n)$, and $f_{21}(\lambda, n)$ are the $(2 N)$ th order polynomials in $\lambda$.

From (2) and (13), we have

$$
a_{n}\left(\lambda_{i}\right)=-\delta_{i, n} b_{n}\left(\lambda_{i}\right), \quad c_{n}\left(\lambda_{i}\right)=-\delta_{i, n} d_{n}\left(\lambda_{i}\right), \quad \delta_{i, n+1}=\frac{-v_{n}+\left(\lambda_{i}-u_{n}\right) \delta_{i, n}}{\delta_{i, n}},
$$


from (23), we can verify that $f_{11}\left(\lambda_{i}, n\right), f_{12}\left(\lambda_{i}, n\right), f_{21}\left(\lambda_{i}, n\right)$, and $f_{22}\left(\lambda_{i}, n\right)$ are all equal to zero. So, $F(\lambda)=\left(\lambda-\lambda_{1}\right)\left(\lambda-\lambda_{2}\right) \cdots\left(\lambda-\lambda_{2 N}\right) P_{n}=\operatorname{det} T_{n} \cdot P_{n}$, where $P_{n}$ is a first-order polynomial. Therefore, we have

$$
T_{n+1} U T_{n}{ }^{*}=\operatorname{det} T_{n} \cdot P_{n},
$$

with

$$
P_{n}=P_{n}^{1} \lambda+P_{n}^{0}
$$

in which $P_{n}^{1}$ and $P_{n}^{0}$ are independent of $\lambda$. Thus, we obtain

$$
T_{n+1} U=P_{n} T_{n}
$$

By use of transformation (20), comparing the coefficients of $\lambda^{N}$ and $\lambda^{N+1}$ in Eq. (26), we have

$$
P_{n}^{1}=\left(\begin{array}{ll}
0 & 0 \\
0 & 1
\end{array}\right), \quad P_{n}^{0}=\left(\begin{array}{cc}
0 & 1 \\
-\widetilde{v}_{n} & -\widetilde{u}_{n}
\end{array}\right) .
$$

From Eqs. (10) and (27), we see that $P_{n}=\widetilde{M}_{n}$.

Next, we will prove that the matrix $\widetilde{W}_{n}$ has the same form as $W_{n}$ under transformations (9) and (20). Let $T_{n}^{-1}=T_{n}^{*} / \operatorname{det} T_{n}$ and

$$
G(\lambda)=\left(T_{n, t}+T_{n} W_{n}\right) T_{n}^{*}=\left(\begin{array}{ll}
g_{11}(\lambda, n) & g_{12}(\lambda, n) \\
g_{21}(\lambda, n) & g_{22}(\lambda, n)
\end{array}\right) .
$$

It can be verified that $g_{11}(\lambda, n)$ is the $(2 N+2)$ th order polynomial in $\lambda$, and $g_{12}(\lambda, n)$, $g_{21}(\lambda, n)$, and $g_{22}(\lambda, n)$ are the $(2 N+1)$ th order polynomials in $\lambda$.

Using (2), (7), and (15), we can obtain

$$
\begin{aligned}
& a_{n, t}\left(\lambda_{i}\right)=-\delta_{i, n, t} b_{n}\left(\lambda_{i}\right)-\delta_{i, n} b_{n, t}\left(\lambda_{i}\right), \quad c_{n, t}\left(\lambda_{i}\right)=-\delta_{i, n, t} d_{n}\left(\lambda_{i}\right)-\delta_{i, n} d_{n, t}\left(\lambda_{i}\right), \\
& \delta_{i, n, t}=-v_{n} \lambda_{i}^{2}-u_{n} v_{n}+\left(\lambda_{i}^{2}-u_{n-1}^{2}-v_{n}-v_{n-1}\right) \delta_{i, n}-\left(\lambda_{i}+u_{n-1}\right) \delta_{i, n}^{2},
\end{aligned}
$$

from which we can verify that $g_{11}\left(\lambda_{i}, n\right), g_{12}\left(\lambda_{i}, n\right), g_{21}\left(\lambda_{i}, n\right)$, and $g_{22}\left(\lambda_{i}, n\right)$ are all equal to zero. So, $G(\lambda)=\left(\lambda-\lambda_{1}\right)\left(\lambda-\lambda_{2}\right) \cdots\left(\lambda-\lambda_{2 N}\right) Q_{n}=\operatorname{det} T_{n} \cdot Q_{n}$, where the elements of the matrix $Q_{n}$ are second-order polynomials in $\lambda$. Moreover, we have

$$
\left(T_{n, t}+T_{n} V_{n}\right) T_{n}^{*}=\operatorname{det} T_{n} \cdot Q_{n}
$$

with

$$
Q_{n}=\left(\begin{array}{cc}
Q_{11}^{(2)} \lambda^{2}+Q_{11}^{(1)} \lambda+Q_{11}^{(0)} & Q_{12}^{(1)} \lambda+Q_{12}^{(0)} \\
Q_{21}^{(1)} \lambda+Q_{21}^{(0)} & Q_{22}^{(1)} \lambda+Q_{22}^{(0)}
\end{array}\right) .
$$

Thus, we obtain

$$
T_{n, t}+T_{n} W_{n}=Q_{n} T_{n}
$$


Using (20) and (32), and comparing the coefficients of $\lambda^{N+1}, \lambda^{N}, \lambda^{N-1}$ in (32), we have

$$
\begin{array}{lll}
Q_{11}^{(2)}=-1, & Q_{11}^{(1)}=1, \quad Q_{11}^{(0)}=\widetilde{u}_{n-1}^{2}+\widetilde{v}_{n-1}, \quad Q_{12}^{(1)}=1, \\
Q_{12}^{(0)}=\widetilde{u}_{n-1}, \quad Q_{21}^{(1)}=-\widetilde{v}_{n}, \quad Q_{21}^{(0)}=-\widetilde{u}_{n} \widetilde{v}_{n}, \quad Q_{22}^{(1)}=1, \quad Q_{22}^{(0)}=\widetilde{v}_{n} .
\end{array}
$$

From (11) and (33), we see that $Q_{n}=\widetilde{W}_{n}$.

Finally, we will prove that the matrix $\widetilde{V W}_{n}$ has the same form as $V_{n}$ under transformations (9) and (20). Let $T_{n}^{-1}=T_{n}^{*} / \operatorname{det} T_{n}$ and

$$
H(\lambda)=\left(T_{n, t}+T_{n} V_{n}\right) T_{n}^{*}=\left(\begin{array}{ll}
h_{11}(\lambda, n) & h_{12}(\lambda, n) \\
h_{21}(\lambda, n) & h_{22}(\lambda, n)
\end{array}\right)
$$

It can be verified that $h_{11}(\lambda, n)$ and $h_{22}(\lambda, n)$ are the $(2 N+3)$ th order polynomials in $\lambda$, and $g_{12}(\lambda, n)$ and $g_{21}(\lambda, n)$ are the $(2 N+2)$ th order polynomials in $\lambda$.

Using (2), (8), and (15), we can obtain

$$
\begin{gathered}
a_{n, t}\left(\lambda_{i}\right)=-\delta_{i, n, t} b_{n}\left(\lambda_{i}\right)-\delta_{i, n} b_{n, t}\left(\lambda_{i}\right), \quad c_{n, t}\left(\lambda_{i}\right)=-\delta_{i, n, t} d_{n}\left(\lambda_{i}\right)-\delta_{i, n} d_{n, t}\left(\lambda_{i}\right), \\
\delta_{i, n, t}=v_{n} \lambda_{i}^{2}+u_{n} v_{n} \lambda_{i}+v_{n}^{2}+v_{n} v_{n+1}+u_{n}^{2} v_{n}-\left(\lambda_{i}^{3}+2 v_{n} \lambda_{i}+u_{n} v_{n}-2 u_{n-1} v_{n-1}\right. \\
\left.-u_{n-2} v_{n-1}-u_{n-1}^{3}\right) \delta_{i, n}+\left(\lambda_{i}^{2}+u_{n-1} \lambda+v_{n-1}+v_{n}+u_{n-1}^{2}\right) \delta_{i, n}^{2},
\end{gathered}
$$

from which we can verify that $h_{11}\left(\lambda_{i}, n\right), h_{12}\left(\lambda_{i}, n\right), h_{21}\left(\lambda_{i}, n\right)$, and $h_{22}\left(\lambda_{i}, n\right)$ are all equal to zero. So, $H(\lambda)=\left(\lambda-\lambda_{1}\right)\left(\lambda-\lambda_{2}\right) \cdots\left(\lambda-\lambda_{2 N}\right) R_{n}=\operatorname{det} T_{n} \cdot R_{n}$, where the elements of the matrix $R_{n}$ are third-order polynomials in $\lambda$. Moreover, we have

$$
\left(T_{n, t}+T_{n} V_{n}\right) T_{n}^{*}=\operatorname{det} T_{n} \cdot R_{n}
$$

with

$$
R_{n}=\left(\begin{array}{cc}
R_{11}^{(3)} \lambda^{3}+R_{11}^{(2)} \lambda^{2}+R_{11}^{(1)} \lambda+R_{11}^{(0)} & R_{12}^{(2)} \lambda^{2}+R_{12}^{(1)} \lambda+R_{12}^{(0)} \\
R_{21}^{(2)} \lambda^{2}+R_{21}^{(1)} \lambda+R_{21}^{(0)} & R_{22}^{(3)} \lambda^{3}+R_{22}^{(2)} \lambda^{2}+R_{22}^{(1)} \lambda+R_{22}^{(0)}
\end{array}\right)
$$

Thus, we obtain

$$
T_{n, t}+T_{n} V_{n}=R_{n} T_{n}
$$

Using (20) and (38), and comparing the coefficients of $\lambda^{N+1}, \lambda^{N}, \lambda^{N-1}$ in (38), we have

$$
\begin{aligned}
& R_{11}^{(3)}=\frac{1}{2}, \quad R_{11}^{(2)}=0, \quad R_{11}^{(1)}=\widetilde{v}_{n}, \\
& R_{11}^{(0)}=-\widetilde{u}_{n-1} \widetilde{v}_{n}-2 \widetilde{u}_{n-1} \widetilde{v}_{n-1}-\widetilde{u}_{n-2} \widetilde{v}_{n-1}-\widetilde{u}_{n-1}^{3}, \quad R_{12}^{(2)}=-1, \quad R_{12}^{(1)}=-\widetilde{u}_{n-1}, \\
& R_{12}^{(0)}=-\widetilde{v}_{n-1}-\widetilde{v}_{n}-\widetilde{u}_{n-1}^{2}, \quad R_{21}^{(2)}=\widetilde{v}_{n}, \quad R_{21}^{(1)}=\widetilde{u}_{n} \widetilde{v}_{n}, \\
& R_{21}^{(0)}=\widetilde{v}_{n}^{2}+\widetilde{v}_{n} \widetilde{v}_{n+1}+\widetilde{u}_{n}^{2} \widetilde{v}_{n}, \quad R_{22}^{(3)}=-\frac{1}{2}, \quad R_{22}^{(2)}=0, \quad Q_{22}^{(1)}=-\widetilde{v}_{n} .
\end{aligned}
$$

From (12) and (39), we see that $R_{n}=\widetilde{V}_{n}$. The proof is completed. 
In fact, Theorem 1 is to prove that matrices $\widetilde{M}_{n}, \widetilde{W}_{n}, \widetilde{V}_{n}$ and $M_{n}, W_{n}, V_{n}$ keep invariant for their forms. The proof is quite similar to the Toda lattice equation in Ref. [35]. From Theorem 1, transformations (9) and (20) can change the Lax pair (2), (7), and (8) into the Lax pair of the same forms (10), (11), and (12). Both of two old and new Lax pairs are compatibility conditions of Eqs. (5) and (6). Therefore, transformations (9) and (20) map the old solutions $u_{n}, v_{n}$ of Eqs. (5) and (6) into the new solutions $\widetilde{u}_{n}, \widetilde{v}_{n}$, we call (9) and (20) the discrete $N$-fold DT of Eqs. (5) and (6). We will emphasize here that the discrete $N$-fold DT (9) and (20) of Eqs. (5) and (6) is the same as the one for the classical Toda lattice equation. As a matter of fact, the same discrete $N$-fold DT can be applied to any member of the Toda hierarchy, and the formula for multi-solitons is the same as the ones in Ref. [35], while there is a big difference among these three discrete $N$-fold DTs that they use different solutions of three Lax pairs. Next let us note that the transformations can be used to determine the multi-soliton solutions of Eq. (5) and (6) when $u_{n}, v_{n}$ are the initial constant solutions.

\section{Dynamical behaviors of multi-soliton solutions of Eq. (5)}

In this part, we will give some multi-dark soliton solutions of Eq. (5) from non-vanishing background via transformations (9) and (20). Substituting the trivial solutions $u_{n}=v_{n}=1$ into the Lax pair (2) and (7), we can give two basic solutions of (2) and (7) with $\lambda=\lambda_{i}$ as follows:

$$
\varphi=\left(\begin{array}{c}
\tau_{1}^{n} e^{\rho_{1} t} \\
\tau_{1}^{n+1} e^{\rho_{1} t}
\end{array}\right), \quad \psi=\left(\begin{array}{c}
\tau_{2}^{n} e^{\rho_{2} t} \\
\tau_{2}^{n+1} e^{\rho_{2} t}
\end{array}\right)
$$

where

$$
\begin{aligned}
\tau_{1} & =\frac{1}{2} \lambda_{i}-\frac{1}{2}+\frac{1}{2} \sqrt{\lambda_{i}^{2}-2 \lambda_{i}-3}, \\
\tau_{2} & =\frac{1}{2} \lambda_{i}-\frac{1}{2}-\frac{1}{2} \sqrt{\lambda_{i}^{2}-2 \lambda_{i}-3}, \\
\rho_{1} & =\left(-\frac{1}{2} \lambda_{i}+\frac{3}{2}+\frac{1}{2} \sqrt{\lambda_{i}^{2}-2 \lambda_{i}-3}\right)\left(\lambda_{i}+1\right), \\
\rho_{2} & =\left(-\frac{1}{2} \lambda_{i}+\frac{3}{2}-\frac{1}{2} \sqrt{\lambda_{i}^{2}-2 \lambda_{i}-3}\right)\left(\lambda_{i}+1\right) .
\end{aligned}
$$

Moreover, taking into account (15), we have

$$
\delta_{i, n}=\frac{\varphi_{2, n}\left(\lambda_{i}\right)-r_{i} \psi_{2, n}\left(\lambda_{i}\right)}{\varphi_{1, n}\left(\lambda_{i}\right)-r_{i} \psi_{1, n}\left(\lambda_{i}\right)}=\frac{\tau_{1}^{n+1} e^{\rho_{1} t}-r_{i} \tau_{2}^{n+1} e^{\rho_{2} t}}{\tau_{1}^{n} e^{\rho_{1} t}-r_{i} \tau_{2}^{n} e^{\rho_{2} t}}, \quad \delta_{i, n+1}=\frac{-1+\left(\lambda_{i}-1\right) \delta_{i, n}}{\delta_{i, n}} .
$$

According to (20) and (41), we can derive the exact solutions of Eq. (5). To understand them, when $N=1,2$, we plot their structure figures as shown in Figs. 1 to 4 .

(I) When $N=1$, let $\lambda=\lambda_{i}(i=1,2)$. Solving the linear algebraic system (14) leads to

$$
b_{n}^{(0)}=\frac{\Delta b_{n}^{(0)}}{\Delta_{1}}, \quad c_{n}^{(0)}=\frac{\Delta c_{n}^{(0)}}{\Delta_{2}}, \quad d_{n}^{(0)}=\frac{\Delta d_{n}^{(0)}}{\Delta_{2}}
$$




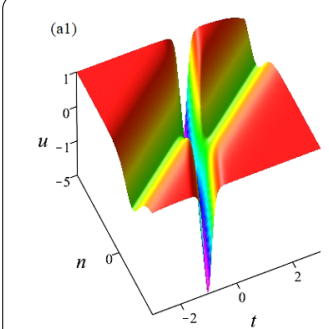

(b1)
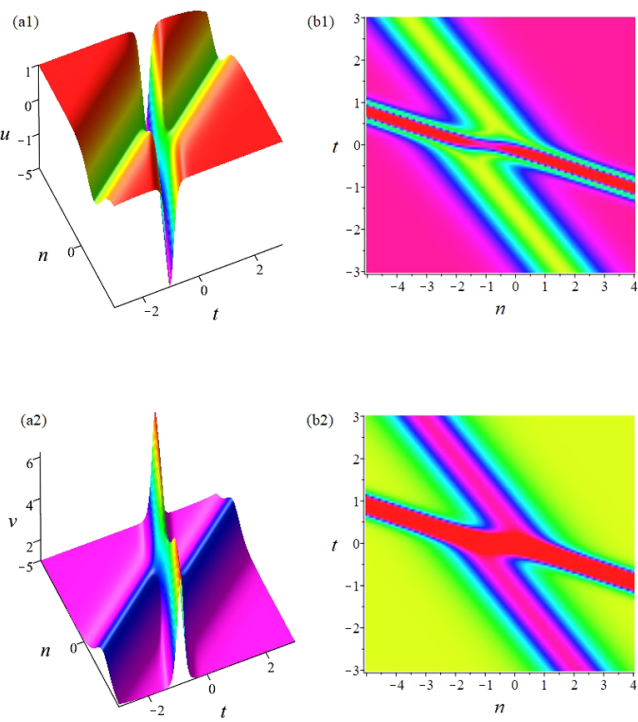

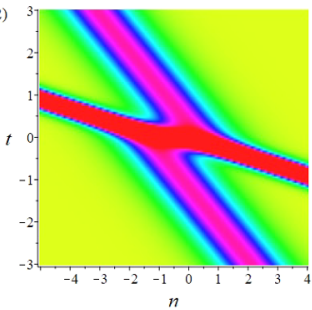

(c1)

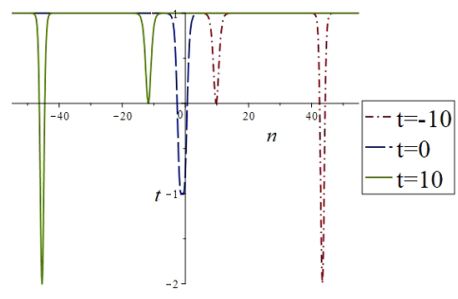

(c2)

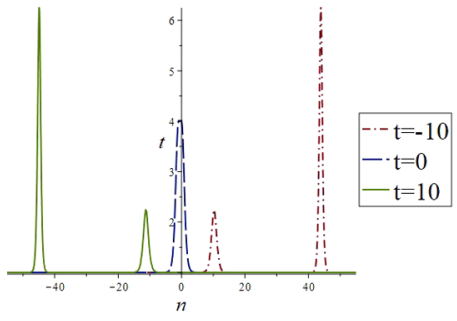

Figure 1 (a1), (b1) Overtaking elastic interactions of dark two-soliton solution $\widetilde{u}_{n}$ in (43) with the parameters $r_{1}=-1, r_{2}=1, \lambda_{1}=-2, \lambda_{2}=-4$. (a2), (b2) Overtaking elastic interactions of bright two-soliton solution $\widetilde{v}_{n}$ in (43) with the same parameters as in (a1), (b1). The interaction processes for (c1) $\widetilde{u}_{n}$ and (c2) $\widetilde{v}_{n}$ at $t=-10$ (dash-dot line), $t=0$ (dashed line), and $t=10$ (solid line)
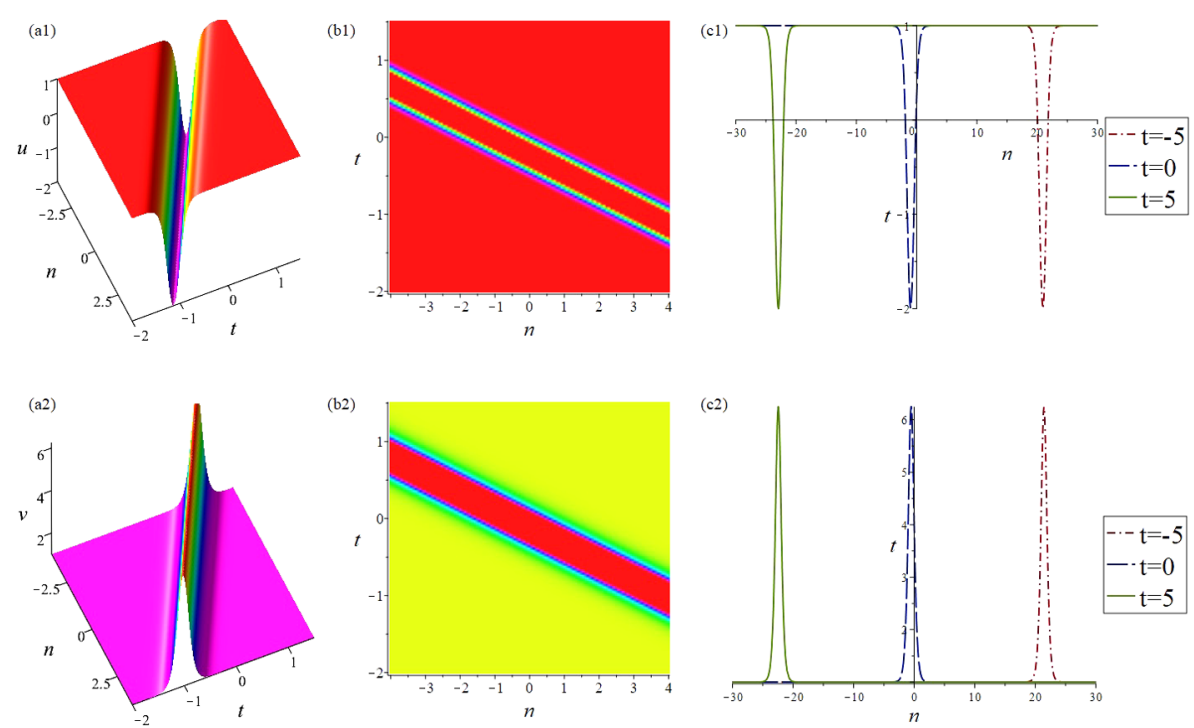

Figure 2 (a1)-(c1) Dark one-soliton solution $\widetilde{u}_{n}$ in (43). (a2)-(c2) Bright one-soliton solution $\widetilde{v}_{n}$ in (43). The same parameters as Fig. 1 except that $\lambda_{1}=-1$

where

$$
\begin{array}{ll}
\Delta b_{n}^{(0)}=\left|\begin{array}{cc}
1 & -\lambda_{1} \\
1 & -\lambda_{2}
\end{array}\right|, \quad \Delta c_{n}^{(0)}=\left|\begin{array}{cc}
-\lambda_{1} \delta_{1, n} & \delta_{1, n} \\
-\lambda_{2} \delta_{2, n} & \delta_{2, n}
\end{array}\right|, \quad \Delta d_{n}^{(0)}=\left|\begin{array}{cc}
1 & -\lambda_{1} \delta_{1, n} \\
1 & -\lambda_{2} \delta_{2, n}
\end{array}\right|, \\
\Delta_{1}=\left|\begin{array}{ll}
1 & \delta_{1, n}-\lambda_{1} \\
1 & \delta_{2, n}-\lambda_{2}
\end{array}\right|, & \Delta_{2}=\left|\begin{array}{ll}
1 & \delta_{1, n} \\
1 & \delta_{2, n}
\end{array}\right| .
\end{array}
$$



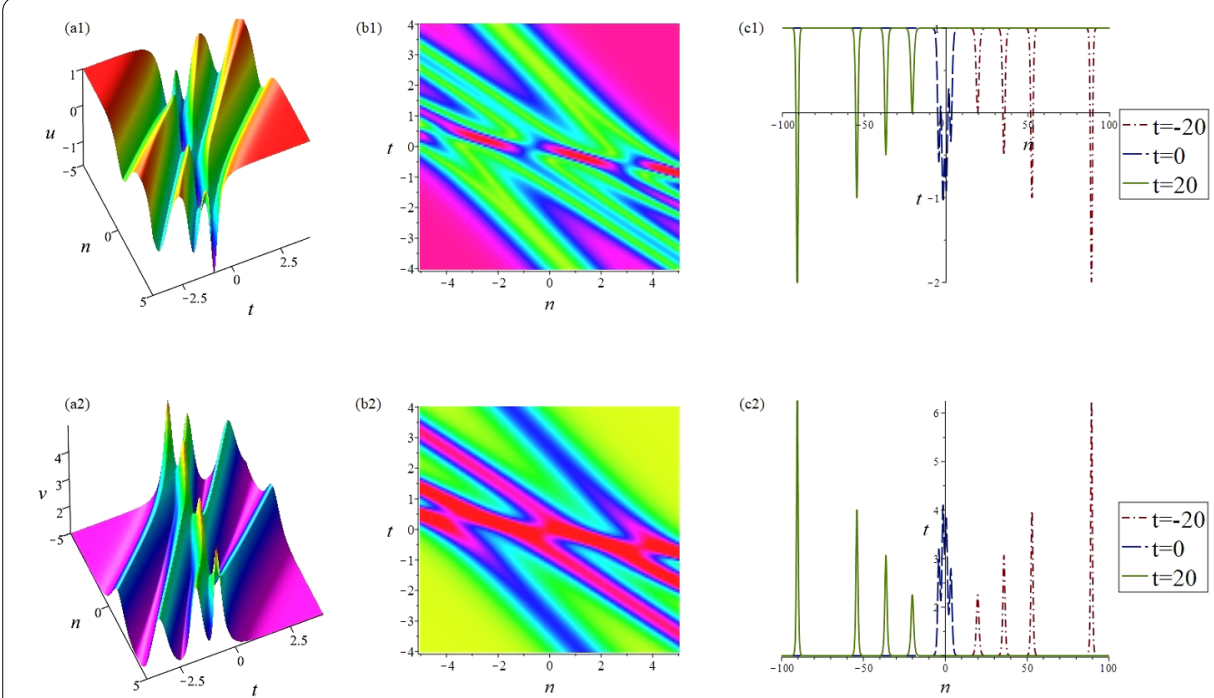

Figure 3 (a1), (b1) Overtaking elastic interactions of dark four-soliton solution $\widetilde{u}_{n}$ in (45) with the parameters $r_{1}=1, r_{2}=-1, r_{3}=\frac{1}{2}, r_{2}=-\frac{1}{2}, \lambda_{1}=-4, \lambda_{2}=-3, \lambda_{3}=-2.5, \lambda_{4}=-2$. (a2), (b2) Overtaking elastic interactions of bright four-soliton solution $\widetilde{v}_{n}$ in (45) with the same parameters as in (a1), (b1). The interaction processes for (c1) $\widetilde{u}_{n}$ and (c2) $\widetilde{v}_{n}$ at $t=-20$ (dash-dot line), $t=0$ (dashed line), and $t=20$ (solid line)
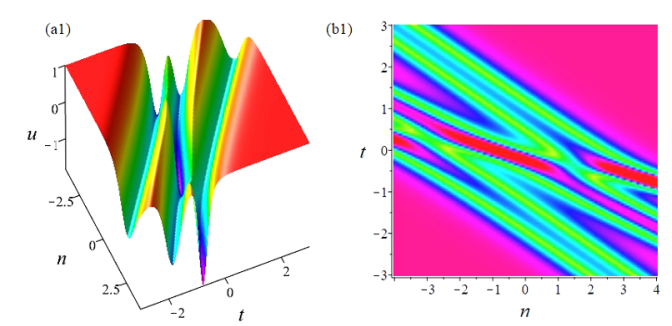

(c1)
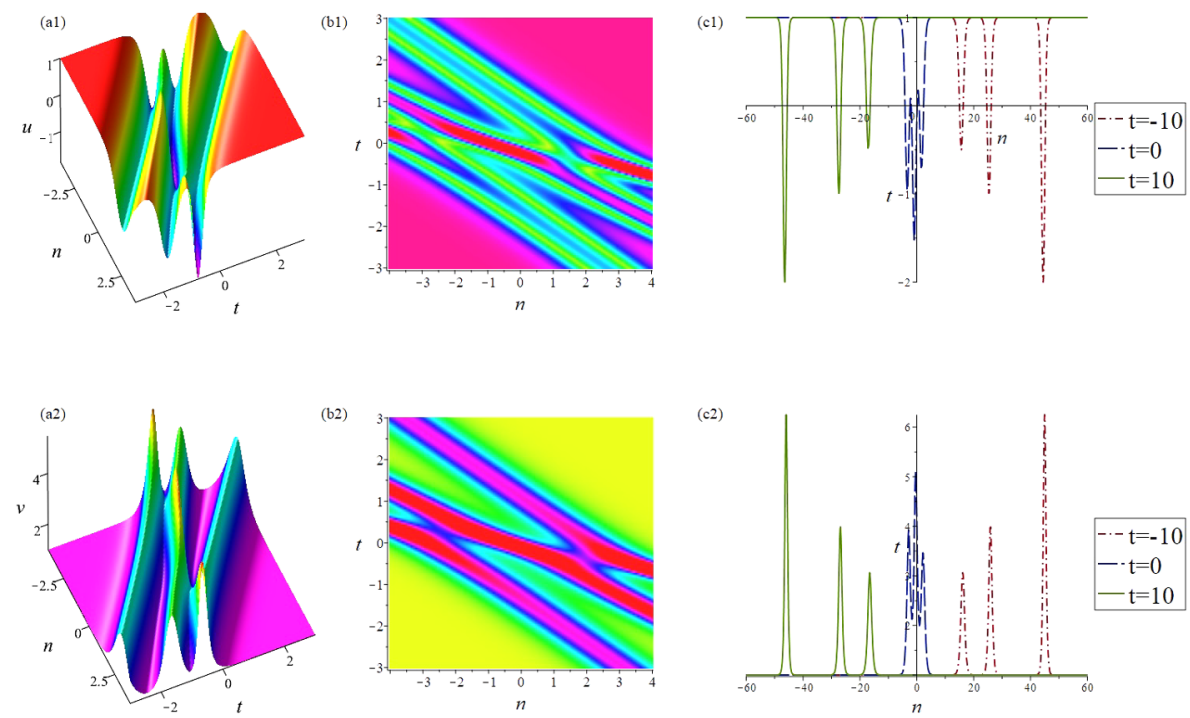

Figure 4 (a1)-(c1) Overtaking elastic interactions of dark three-soliton solution $\widetilde{u}_{n}$ in (45). (a2)-(c2) Overtaking elastic interactions of bright four-soliton solution $\widetilde{v}_{n}$ in (45) with the same parameters as in (a1)-(c1). The same parameters as Fig. 3 except that $\lambda_{4}=-1$

Consequently, an explicit 1-fold exact solution of (5) can be written via the discrete 1-fold DT as follows:

$$
\widetilde{u}_{n}=1+d_{n}^{(0)}-d_{n+1}^{(0)}, \quad \widetilde{v}_{n}=\frac{1+c_{n}^{(0)}}{1-b_{n}^{(0)}}
$$

When $\lambda_{1} \geq 3, \lambda_{2} \geq 3$ or $\lambda_{1} \leq-1, \lambda_{2} \leq-1$ or $\lambda_{1} \geq 3, \lambda_{2} \leq-1$ or $\lambda_{1} \leq-1, \lambda_{2} \geq 3$ and the parameters $r_{i}(i=1,2)$ are real and not equal to zero, solution (43) may be the one-soliton 
or two-soliton solution, the corresponding evolution plots are shown in Figs. 1 and 2 . Figures 1(a1)-(c1) show the overtaking interactions between two unidirectional anti-bellshape dark solitons with different amplitudes for solution $u_{n}$, the soliton with a larger amplitude moves faster and overtakes the smaller, the amplitude minimum for the smaller soliton is 0 , the amplitude minimum for the larger soliton is -2 , however the background level here is 1 . Thus, the minimums of the smaller and larger amplitudes are 1 and 3 units lower than the background. At last, the shapes of two dark solitons do not change before and after the interaction, their interactions are elastic. Figures $1(\mathrm{a} 2)-(\mathrm{c} 2)$ reveal the overtaking interactions between two unidirectional bell-shape bright solitons with different amplitudes for solution $v_{n}$, the soliton with a higher amplitude moves faster and overtakes the lower, the maximal amplitude of the high soliton is 6.25 , the maximal amplitude of the low soliton is 2.25 , however the background level here is 1 . Thus, the maximums of the high and low amplitudes are 1.25 and 5.25 units higher than the background. After interaction, the final bright solitons preserve their shapes and amplitudes, propagate along the negative $n$-direction, and their interactions are elastic. However, it is worthwhile to note that the above two solitons will reduce to one soliton when one of two $\lambda$ 's is equal to -1 . For $\lambda_{1}$ and $\lambda_{2}$ when $N=1$, suppose $\lambda_{1}=-1$, we can see that $\tau_{1}=\tau_{2}=-\frac{3}{2}, \rho_{1}=\rho_{2}=0$ in (40), while $\delta_{1, n}=-\frac{3}{2}$ in (42) and (43). That is to say, the two solutions of the Lax pair are linearly dependent. At this point, solution (43) only uses one solution of the Lax pair (2) and (7), so the previous two solitons will reduce to one, while solution (43) uses two different linearly independent solutions of the Lax pair when $\lambda_{1} \neq-1$, which will lead to two solitons. Figures 2(a1)-(c1) present the evolution structures of the anti-bell-shape dark one-soliton solution $u_{n}$, the minimal amplitude of the solution is along a line, the minimum is -2 , however the background level here is 1 . Thus, the minimum is 3 units lower than the background. Figures 2(a2)-(c2) show the evolution structures of the bell-shape bright one-soliton solution $v_{n}$, the maximal amplitude of the solution is also along a line, the maximum is 6.25 , however the background level here is also 1 . Thus, the maximum is 5.25 units higher than the background. As shown in Figs. 2, both one-soliton solutions $u_{n}$ and $v_{n}$ propagate along the negative $n$-axis direction with the same amplitude, and their shapes remain the same during the propagation.

(II) When $N=2$, let $\lambda=\lambda_{i}(i=1,2,3,4)$. Solving the linear algebraic system (14) gives

$$
b_{n}^{(1)}=\frac{\Delta b_{n}^{(1)}}{\Delta_{1}}, \quad c_{n}^{(1)}=\frac{\Delta c_{n}^{(1)}}{\Delta_{2}}, \quad d_{n}^{(1)}=\frac{\Delta d_{n}^{(1)}}{\Delta_{2}},
$$

where

$$
\begin{aligned}
\Delta b_{n}^{(1)} & =\left|\begin{array}{llll}
1 & \lambda_{1} & \delta_{1, n} & -\lambda_{1}^{2} \\
1 & \lambda_{2} & \delta_{2, n} & -\lambda_{2}^{2} \\
1 & \lambda_{3} & \delta_{3, n} & -\lambda_{3}^{2} \\
1 & \lambda_{4} & \delta_{4, n} & -\lambda_{4}^{2}
\end{array}\right|, \quad \Delta c_{n}^{(1)}=\left|\begin{array}{cccc}
1 & -\lambda_{1}^{2} \delta_{1, n} & \delta_{1, n} & \lambda_{1} \delta_{1, n} \\
1 & -\lambda_{2}^{2} \delta_{2, n} & \delta_{2, n} & \lambda_{2} \delta_{2, n} \\
1 & -\lambda_{3}^{2} \delta_{3, n} & \delta_{3, n} & \lambda_{3} \delta_{3, n} \\
1 & -\lambda_{4}^{2} \delta_{4, n} & \delta_{4, n} & \lambda_{4} \delta_{4, n}
\end{array}\right|, \\
\Delta d_{n}^{(1)} & =\left|\begin{array}{lllll}
1 & \lambda_{1} & \delta_{1, n} & -\lambda_{1}^{2} \delta_{1, n} \\
1 & \lambda_{2} & \delta_{2, n} & -\lambda_{2}^{2} \delta_{2, n} \\
1 & \lambda_{3} & \delta_{3, n} & -\lambda_{3}^{2} \delta_{3, n} \\
1 & \lambda_{4} & \delta_{4, n} & -\lambda_{4}^{2} \delta_{4, n}
\end{array}\right|, \quad \Delta_{1}=\left|\begin{array}{llll}
1 & \lambda_{1} & \delta_{1, n} & \lambda_{1} \delta_{1, n}-\lambda_{1}^{2} \\
1 & \lambda_{2} & \delta_{2, n} & \lambda_{2} \delta_{2, n}-\lambda_{2}^{2} \\
1 & \lambda_{3} & \delta_{3, n} & \lambda_{3} \delta_{3, n}-\lambda_{3}^{2} \\
1 & \lambda_{4} & \delta_{4, n} & \lambda_{4} \delta_{4, n}-\lambda_{4}^{2}
\end{array}\right|,
\end{aligned}
$$




$$
\Delta_{2}=\left|\begin{array}{cccc}
1 & \lambda_{1} & \delta_{1, n} & \lambda_{1} \delta_{1, n} \\
1 & \lambda_{2} & \delta_{2, n} & \lambda_{2} \delta_{2, n} \\
1 & \lambda_{3} & \delta_{3, n} & \lambda_{3} \delta_{3, n} \\
1 & \lambda_{4} & \delta_{4, n} & \lambda_{4} \delta_{4, n}
\end{array}\right| .
$$

Thus, via the discrete 2-fold DT, the 2-fold explicit exact solution of (5) can be written as

$$
\widetilde{u}_{n}=1+d_{n}^{(1)}-d_{n+1}^{(1)}, \quad \widetilde{v}_{n}=\frac{1+c_{n}^{(1)}}{1-b_{n}^{(1)}}
$$

When $\lambda_{i} \geq 3(i=1,2,3,4)$ or $\lambda_{i} \leq-1(i=1,2,3,4)$ and the parameters $r_{i}(i=1,2)$ are real and not equal to zero, solution (45) with $N=2$ may be the three or four solitons, the corresponding evolution plots are shown in Figs. 3 and 4. Figures 3(a1)-(c1) show the overtaking collision interactions among four unidirectional anti-bell-shape dark solitons with different amplitudes for solution $\widetilde{u}_{n}$ in (45), the amplitude minimums of the four dark solitons are around $0,-0.5,-1$, and 2 , respectively, however the background level here is 1. Thus, the minimums are 1, 1.5, 2, and 3 units under the background. Figures 3(a2)-(c2) show the overtaking collision interactions among four bell-shape unidirectional bright solitons with different amplitudes for solution $\widetilde{v}_{n}$ in (45), the amplitudes of the four bright solitons are around $2.25,3,4$, and 6.25 , respectively, however the background level here is 1. Thus, the maximums are 1.25, 2, 3, and 5.25 units above the background. However, it is worthwhile to note that this solution is the three-soliton solution if when one of four $\lambda$ 's is equal to -1 . Figures $4(\mathrm{a} 1)-(\mathrm{c} 1)$ present the overtaking collision interactions among three unidirectional anti-bell-shape dark solitons with different amplitudes for solution $\widetilde{u}_{n}$ in (45), the minimums of amplitude for the three dark solitons are 1.5, 2, and 3 units under the background level 1, respectively. Figures 4(a2)-(c2) reveal the overtaking collision interactions among three unidirectional bell-shape bright solitons with different amplitudes for solution $\widetilde{u}_{n}$ in (45), the maximums of amplitude for the three bright solitons are 2, 3, and 5.25 units, respectively, above the background level 1. Before and after the overtaking interactions, the final three and four solitons preserve their shapes and amplitudes and move along the negative $n$-axis direction, so their interactions are elastic.

(III) When $N=m$, let $\lambda=\lambda_{i}(i=1,2, \ldots, 2 m)$. Solving the linear algebraic system (14) leads to

$$
b_{n}^{(m-1)}=\frac{\Delta b_{n}^{(m-1)}}{\Delta_{1}}, \quad c_{n}^{(m-1)}=\frac{\Delta c_{n}^{(m-1)}}{\Delta_{2}}, \quad d_{n}^{(m-1)}=\frac{\Delta d_{n}^{(m-1)}}{\Delta_{2}},
$$

where

$$
\begin{aligned}
\Delta b_{n}^{(m-1)} & =\left|\begin{array}{cccccccc}
1 & \lambda_{1} & \cdots & \lambda_{1}^{m-1} & \delta_{1, n} & \lambda_{1} \delta_{1, n} & \cdots & -\lambda_{1}^{m} \\
1 & \lambda_{2} & \cdots & \lambda_{2}^{m-1} & \delta_{2, n} & \lambda_{2} \delta_{2, n} & \cdots & -\lambda_{2}^{m} \\
\cdots & \cdots & \cdots & \cdots & \cdots & \cdots & \cdots & \cdots \\
1 & \lambda_{2 m} & \cdots & \lambda_{2 m}^{m-1} & \delta_{2 m, n} & \lambda_{2 m} \delta_{2 m, n} & \cdots & \lambda_{2 m}^{m}
\end{array}\right|, \\
\Delta c_{n}^{(m-1)} & =\left|\begin{array}{cccccccc}
1 & \lambda_{1} & \cdots & -\delta_{1, n} \lambda_{1}^{m} & \delta_{1, n} & \lambda_{1} \delta_{1, n} & \cdots & \lambda_{1}^{m-1} \delta_{1, n} \\
1 & \lambda_{2} & \cdots & -\delta_{2, n} \lambda_{2}^{m} & \delta_{2, n} & \lambda_{2} \delta_{2, n} & \cdots & \lambda_{2}^{m-1} \delta_{2, n} \\
\cdots & \cdots & \cdots & \cdots & \cdots & \cdots & \cdots & \cdots \\
1 & \lambda_{2 m} & \cdots & -\delta_{2 m, n} \lambda_{2 m}^{m} & \delta_{2 m, n} & \lambda_{2 m} \delta_{2 m, n} & \cdots & \lambda_{2 m}^{m-1} \delta_{2 m, n}
\end{array}\right|,
\end{aligned}
$$




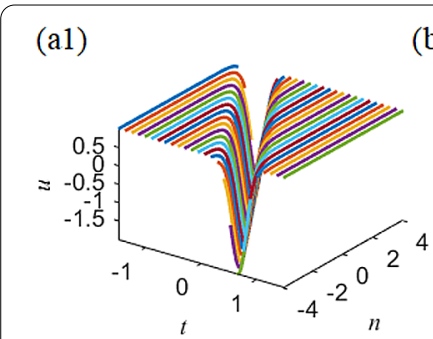

(a2)

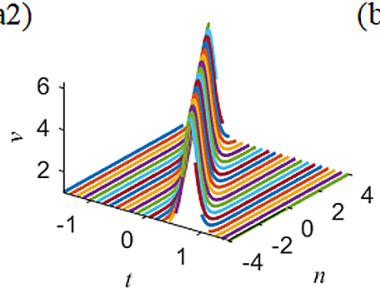

(b1)

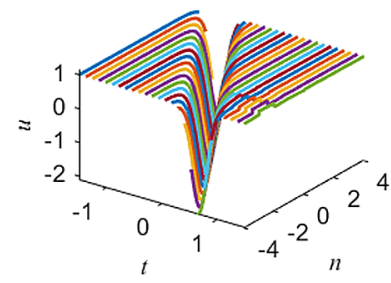

(b2)

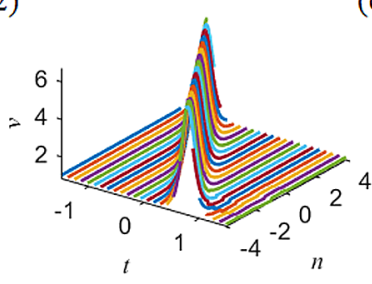

(c1)

$=-1$

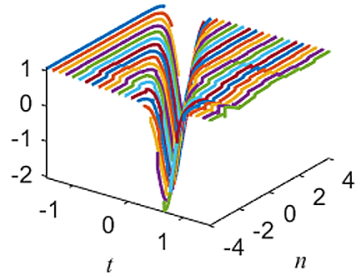

c2)

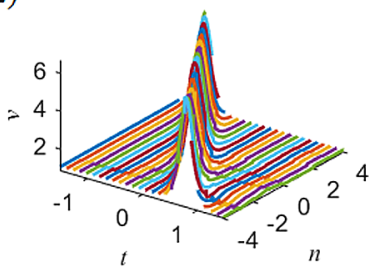

Figure 5 One-soliton solution (43) with the same parameters as Fig. 2. (a) Exact solution; (b) Time evolution using exact solution (43) as an initial condition; (c) Time evolution using exact solution (43) perturbed by a 5\% noise as the initial condition

$$
\begin{gathered}
\Delta d_{n}^{(m-1)}=\left|\begin{array}{cccccccc}
1 & \lambda_{1} & \cdots & \lambda_{1}^{m-1} & \delta_{1, n} & \lambda_{1} \delta_{1, n} & \cdots & -\delta_{1, n} \lambda_{1}^{m} \\
1 & \lambda_{2} & \cdots & \lambda_{2}^{m-1} & \delta_{2, n} & \lambda_{2} \delta_{2, n} & \cdots & -\delta_{2, n} \lambda_{2}^{m} \\
\cdots & \cdots & \cdots & \cdots & \cdots & \cdots & \cdots & \cdots \\
1 & \lambda_{2 m} & \cdots & \lambda_{2 m}^{m-1} & \delta_{2 m, n} & \lambda_{2 m} \delta_{2 m, n} & \cdots & -\delta_{2 m, n} \lambda_{2 m}^{m}
\end{array}\right|, \\
\Delta_{1}=\left|\begin{array}{cccccccc}
1 & \lambda_{1} & \cdots & \lambda_{1}^{m-1} & \delta_{1, n} & \lambda_{1} \delta_{1, n} & \cdots & \lambda_{1}^{m-1} \delta_{1, n}-\lambda_{1}^{m} \\
1 & \lambda_{2} & \cdots & \lambda_{2}^{m-1} & \delta_{2, n} & \lambda_{2} \delta_{2, n} & \cdots & \lambda_{2}^{m-1} \delta_{2, n}-\lambda_{2}^{m} \\
\cdots & \cdots & \cdots & \cdots & \cdots & \cdots & \cdots & \cdots \\
1 & \lambda_{2 m} & \cdots & \lambda_{2 m}^{m-1} & \delta_{2 m, n} & \lambda_{2 m} \delta_{2 m, n} & \cdots & \lambda_{2 m}^{m-1} \delta_{2 m, n}-\lambda_{2 m}^{m}
\end{array}\right|, \\
\Delta_{2}=\left|\begin{array}{cccccccc}
1 & \lambda_{1} & \cdots & \lambda_{1}^{m-1} & \delta_{1, n} & \lambda_{1} \delta_{1, n} & \cdots & \lambda_{1}^{m-1} \delta_{1, n} \\
1 & \lambda_{2} & \cdots & \lambda_{2}^{m-1} & \delta_{2, n} & \lambda_{2} \delta_{2, n} & \cdots & \lambda_{2}^{m-1} \delta_{2, n} \\
\cdots & \cdots & \cdots & \cdots & \cdots & \cdots & \cdots & \cdots \\
1 & \lambda_{2 m} & \cdots & \lambda_{2 m}^{m-1} & \delta_{2 m, n} & \lambda_{2 m} \delta_{2 m, n} & \cdots & \lambda_{2 m}^{m-1} \delta_{2 m, n}
\end{array}\right| .
\end{gathered}
$$

In this way, a general $m$-fold explicit exact solution of Eq. (5) can be obtained via the $m$-fold DT written as follows:

$$
\widetilde{u}_{n}=1+d_{n}^{(m-1)}-d_{n+1}^{(m-1)}, \quad \widetilde{v}_{n}=\frac{1+c_{n}^{(m-1)}}{1-b_{n}^{(m-1)}},
$$

where $d_{n+1}^{(m-1)}$ is derived from $d_{n}^{(m-1)}$ by replacing their $n$ with $n+1$.

Next, we implement the numerical simulations to display the dynamical behaviors of the previous bright and dark multi-solitons of Eq. (5) by using the finite difference method [56, 57]. Figure 5 exhibits the exact one-soliton solution (43) of Eq. (5), time evolutions of using exact one-soliton solution (43), and those perturbed by a small noise $5 \%$. Figures 5(a1), (b1)-(a2), (b2) show that the profiles of the time evolution of the bright and dark onesoliton solution (43) without a noise almost agrees with the ones of the corresponding exact bright and dark one-soliton solution (43) in time $t \in(-1.5,1.5)$. It is clearly seen that our numerical solutions exactly reproduce the analytical solutions; in other words, 
these solutions have the stable evolutions without a noise, which also shows the accuracy of our numerical scheme. We further numerically study the stability of these solutions by perturbing the above initial conditions (i.e., a small noise (5\%) is added to both the initial exact solutions $u_{n}$ and $v_{n}$ components with $t=-1.5$ ), the numerical results in Figs. 5(c1)(c2) show that they can evolve as before, that is to say, one-soliton solution (43) of Eq. (5) is robust against a small noise.

Figures 6-8 demonstrate the exact two-, three-, and four-soliton solutions of Eq. (5), time evolutions of using their exact solutions, and those perturbed by a small noise $5 \%$, respectively. Just like the one-soliton solution, Figs. 6-8(a1), (b1)-(a2), (b2) show the accuracy of our numerical scheme and the stable evolutions of the two-, three-, and foursoliton solutions without a noise. Numerical results in Figs. 6-8(c1)-(c2) reveal the almost

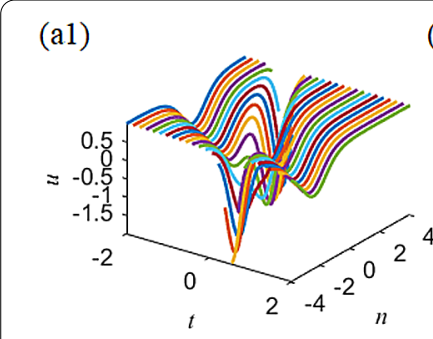

(a2)

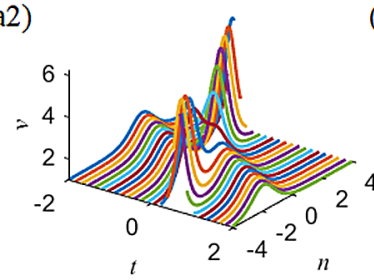

(b1)

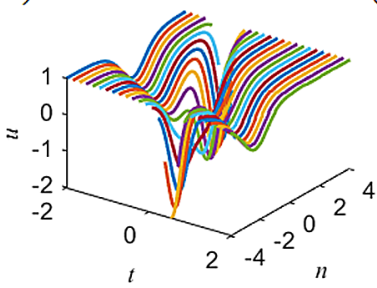

(b2)

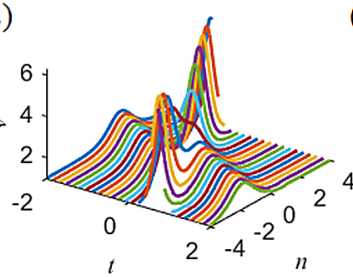

(c1)

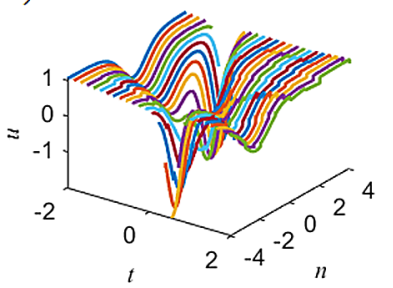

(c2)

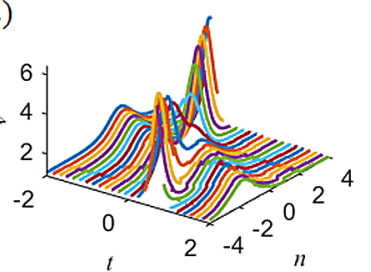

Figure 6 Two-soliton solution (43) with the same parameters as Fig. 1. (a) Exact solution; (b) Time evolution using exact solution (43) as an initial condition; (c) Time evolution using exact solution (43) perturbed by a 5\% noise as the initial condition

(a1)

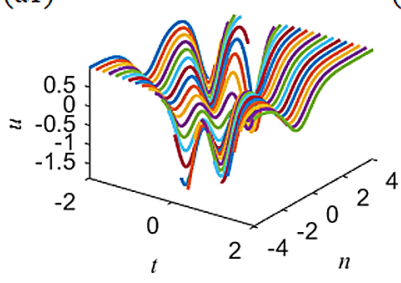

(a2)

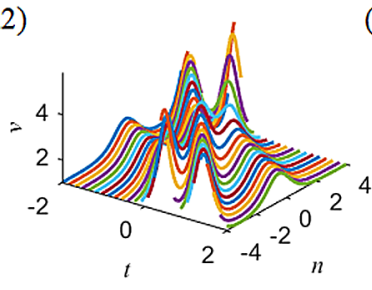

(b1)

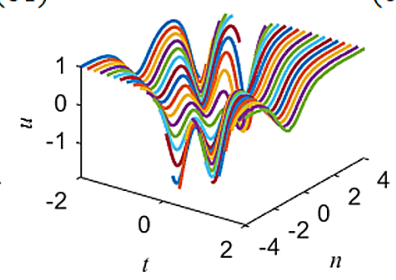

(c1)

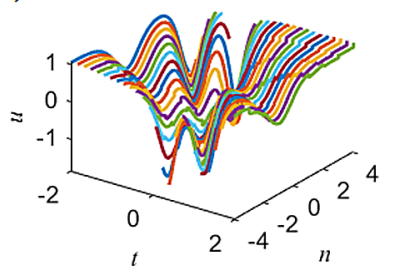

(b2)

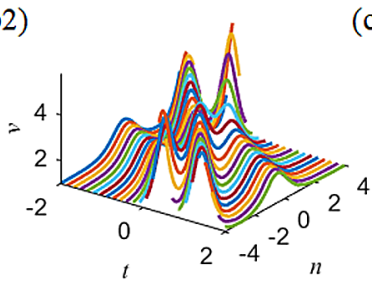

(c2)

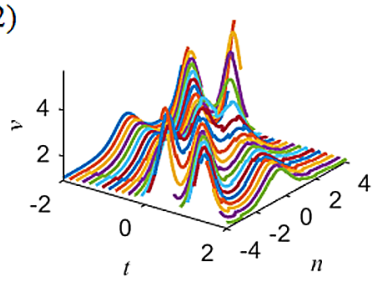

Figure 7 Three-soliton solution (45) with the same parameters as Fig. 4. (a) Exact solution; (b) Time evolution using exact solution (45) as an initial condition; (c) Time evolution using exact solution (45) perturbed by a 5\% noise as the initial condition 


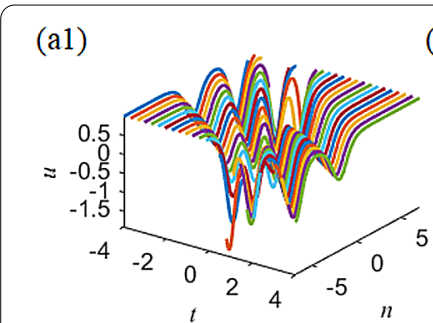

(a2)

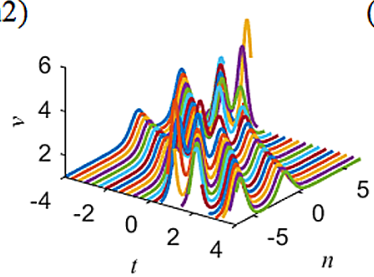

(b1)

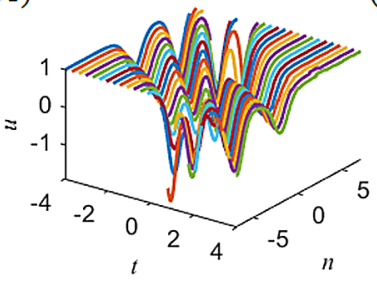

(b2)

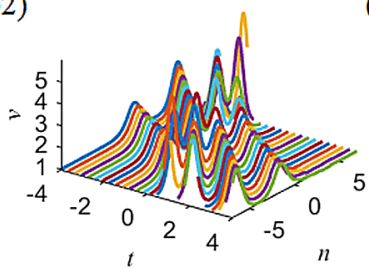

(c1)

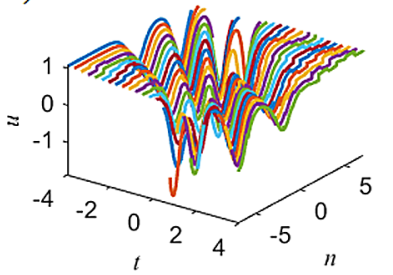

(c2)

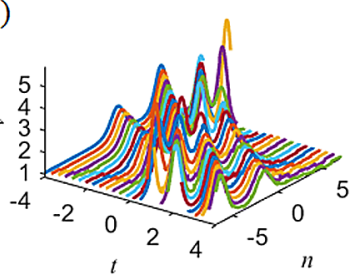

Figure 8 Four-soliton solution (45) with the same parameters as Fig. 3. (a) Exact solution; (b) Time evolution using exact solution (45) as an initial condition; (c) Time evolution using exact solution (45) perturbed by a 5\% noise as the initial condition

steady evolutions of the two-, three-, and four-soliton solutions with a small noise; in other words, these solutions are stable and also robust against a small noise.

\section{Dynamics of multi-soliton solutions of Eq. (6) via numerical simulations}

In this part, we will give some dark and bright soliton solutions of Eq. (6) from nonvanishing background via transformations (9) and (20). Substituting the trivial solutions $u_{n}=v_{n}=1$ into the Lax pair (2) and (8), we can give two basic solutions of (2) and (8) with $\lambda=\lambda_{i}$ as follows:

$$
\varphi=\left(\begin{array}{c}
\tau_{1}^{n} e^{\rho_{1} t} \\
\tau_{1}^{n+1} e^{\rho_{1} t}
\end{array}\right), \quad \psi=\left(\begin{array}{c}
\tau_{2}^{n} e^{\rho_{2} t} \\
\tau_{2}^{n+1} e^{\rho_{2} t}
\end{array}\right),
$$

where

$$
\begin{aligned}
& \tau_{1}=\frac{1}{2} \lambda_{i}-\frac{1}{2}+\frac{1}{2} \sqrt{\lambda_{i}^{2}-2 \lambda_{i}-3}, \\
& \tau_{2}=\frac{1}{2} \lambda_{i}-\frac{1}{2}-\frac{1}{2} \sqrt{\lambda_{i}^{2}-2 \lambda_{i}-3}, \\
& \rho_{1}=-\frac{7}{2}-\frac{1}{2} \sqrt{\lambda^{6}-14 \lambda^{3}-24 \lambda^{2}-36 \lambda-27}, \\
& \rho_{2}=-\frac{7}{2}+\frac{1}{2} \sqrt{\lambda^{6}-14 \lambda^{3}-24 \lambda^{2}-36 \lambda-27} .
\end{aligned}
$$

Moreover, taking into account (15), we have

$$
\delta_{i, n}=\frac{\varphi_{2, n}\left(\lambda_{i}\right)-r_{i} \psi_{2, n}\left(\lambda_{i}\right)}{\varphi_{1, n}\left(\lambda_{i}\right)-r_{i} \psi_{1, n}\left(\lambda_{i}\right)}=\frac{\tau_{1}^{n+1} e^{\rho_{1} t}-r_{i} \tau_{2}^{n+1} e^{\rho_{2} t}}{\tau_{1}^{n} e^{\rho_{1} t}-r_{i} \tau_{2}^{n} e^{\rho_{2} t}}, \quad \delta_{i, n+1}=\frac{-1+\left(\lambda_{i}-1\right) \delta_{i, n}}{\delta_{i, n}} .
$$

According to (20) and (49), we can obtain the explicit exact solution of Eq. (6) as follows:

$$
\widetilde{u}_{n}=1+d_{n}^{(N-1)}-d_{n+1}^{(N-1)}, \quad \widetilde{v}_{n}=\frac{1+c_{n}^{(N-1)}}{1-b_{n}^{(N-1)}},
$$



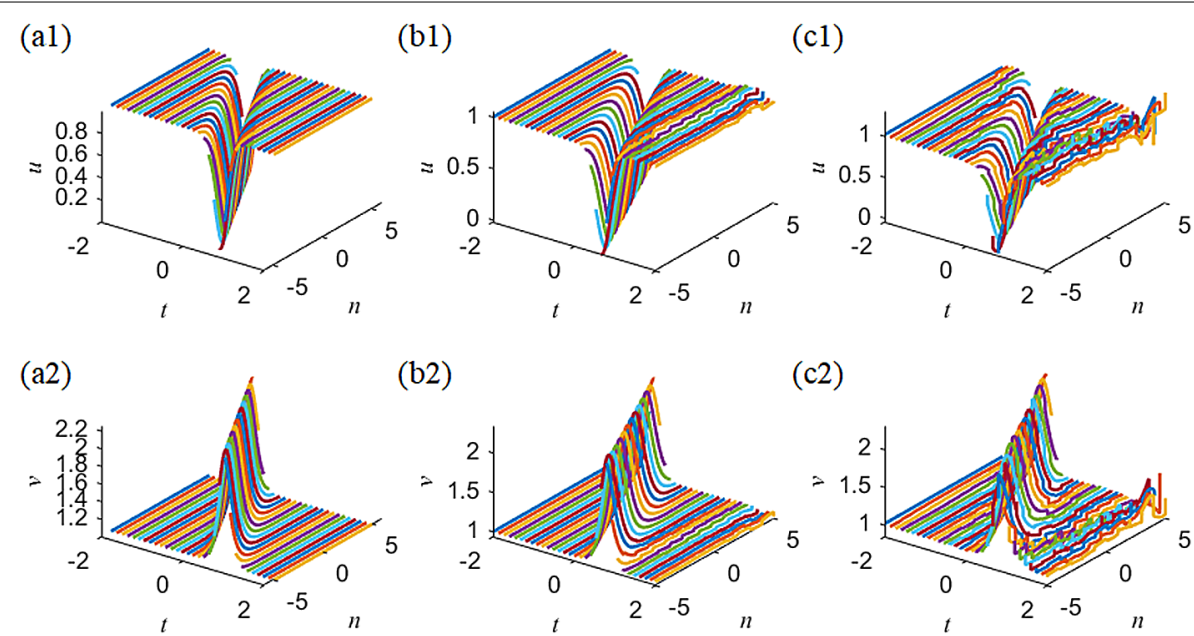

(b2)

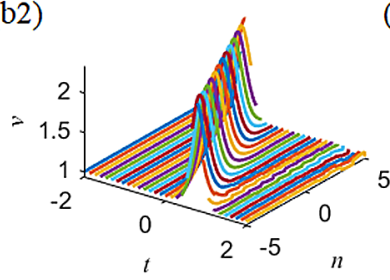

(c2)

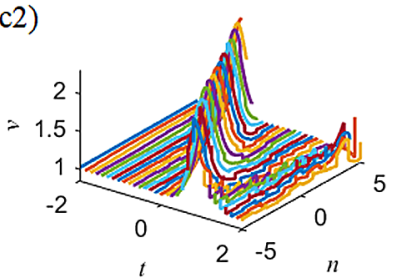

Figure 9 Exact one-soliton solutions $\widetilde{u}_{n}$ and $\widetilde{v}_{n}$ in (50) with $N=1$ via the parameters $r_{1}=-1, r_{2}=1, \lambda_{1}=-1$, $\lambda_{2}=-2$. (a) Exact solution; (b) Time evolution using exact solution as an initial condition; (c) Time evolution using exact solution perturbed by a $2 \%$ noise as the initial condition

where $b_{n}^{(N-1)}, c_{n}^{(N-1)}$, and $d_{n}^{(N-1)}$ are the same as those in the previous section, so we omit their expressions here. The soliton interactions and structures of Eq. (6) are similar to Eq. (5), so the next thing we do is we only perform the numerical simulations to show the dynamical behaviors of the multi-solitons of Eq. (6). Figure 9 exhibits the exact onesoliton solution (50) with $N=1$, time evolutions of using the exact one-soliton solution, and those perturbed by a small noise $2 \%$. Figures $9(\mathrm{a} 1),(\mathrm{b} 1)-(\mathrm{a} 2),(\mathrm{b} 2)$ show that the time evolution profiles of the bright and dark one-soliton solution (50) with $N=1$ without a noise almost agree with the ones of the corresponding exact bright and dark one-soliton solution in time $t \in(-2,2)$. It is clearly seen that our numerical solutions almost exactly reproduce the analytical solutions; in other words, these solutions have the stable evolutions without a noise, which also shows the accuracy of our numerical scheme. We further numerically study the stability of these solutions by perturbing the above initial conditions (i.e., a small noise $(2 \%)$ is added to both the initial exact solutions $u_{n}$ and $v_{n}$ components with $t=-2)$, the numerical results in Figs. 9(c1)-(c2) show the almost steady evolutions as before, in addition to some small oscillations as time close to 2 . With the noise increasing (for example, a small noise 5\% is added to the initial solution), the simulated evolutions exhibit obviously strong oscillations and instability. Compared with Eq. (5), we find that the same small noise has different effect on the evolutions of the soliton solutions for Eqs. (5) and (6). The evolutions of the lower-order equation can keep better propagation stability under a bigger small noise, however the evolutions of the higher-order equation only have the propagation stability under a very small noise. For the higher-order equation, the simulated evolutions exhibit obviously strong oscillations and instability with the noise increasing (for example, adding a 5\% noise), so we infer that the possible reason is that the higher-order nonlinear terms for Eq. (6) cause the propagation instability of the solitons relative to Eq. (5).

Figures 10-12 exhibit the exact two-, three-, and four-soliton solutions of Eq. (6), time evolutions of using their exact solutions, and those perturbed by a small noise $2 \%$, respectively. Like its one-soliton solution, Figs. 10-12(a1), (b1)-(a2), (b2) show the accuracy of our numerical scheme and the almost steady evolutions of the two-, three-, and 


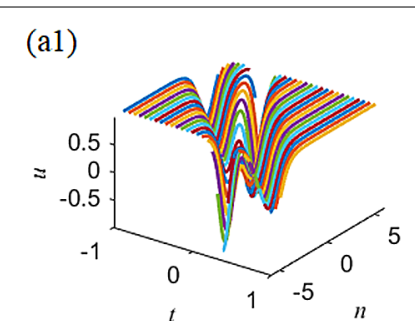

(a2)

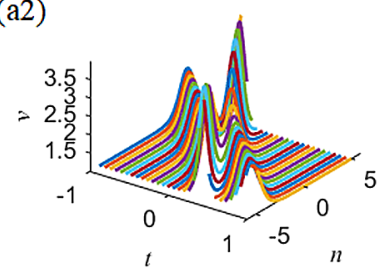

(b1)

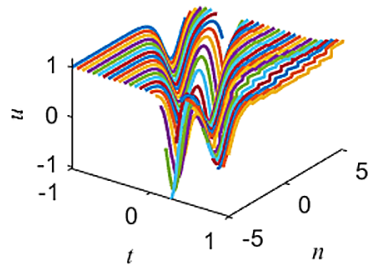

(b2)

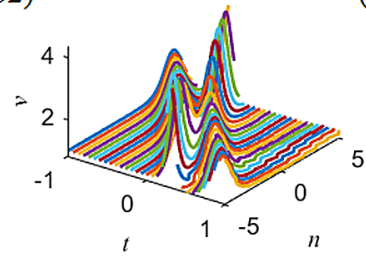

(c1)

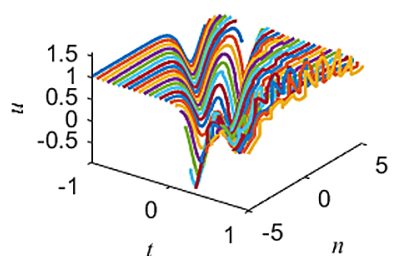

(c2)

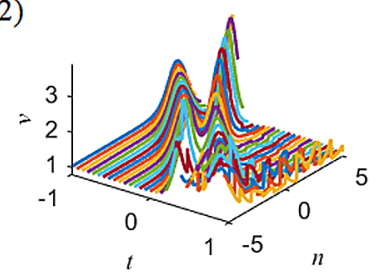

Figure 10 Collisions of two-soliton solutions $\widetilde{u}_{n}$ and $\widetilde{v}_{n}$ in (50) with $N=1$ via the parameters $r_{1}=-1, r_{2}=1$, $\lambda_{1}=-2, \lambda_{2}=-3$. (a) Exact solution; (b) Time evolution using exact solution as an initial condition; (c) Time evolution using exact solution perturbed by a $2 \%$ noise as the initial condition

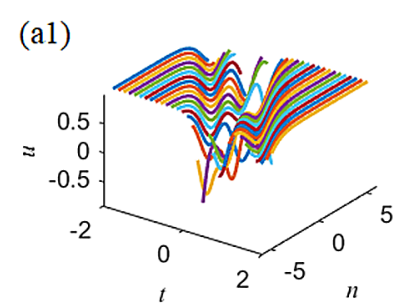

(b1)

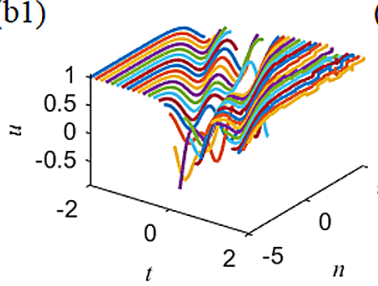

(b2)

(a2)

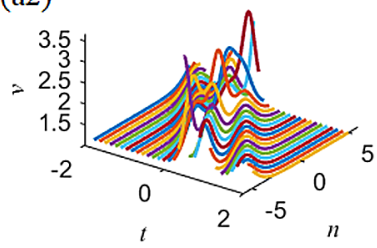

(c1)

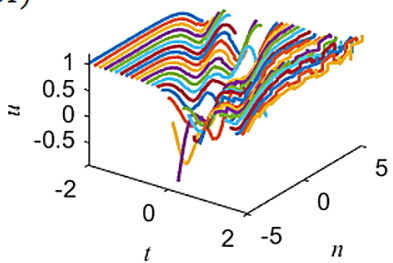

(c2)

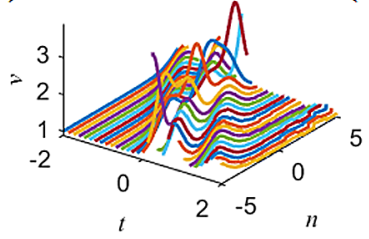

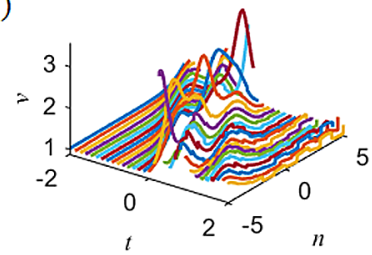

Figure 11 Collisions of three-soliton solutions $\widetilde{u}_{n}$ and $\widetilde{v}_{n}$ in (50) with $N=2$ via the parameters $r_{1}=1, r_{2}=-1$, $r_{3}=1 / 2, r_{4}=-1 / 2, \lambda_{1}=-3, \lambda_{2}=-2, \lambda_{3}=-3 / 2, \lambda_{4}=-1$. (a) Exact solution; (b) Time evolution using exact solution as an initial condition; (c) Time evolution using exact solution perturbed by a $2 \%$ noise as the initial condition

four-soliton solutions without a noise. Numerical results in Figs. 10-12(c1)-(c2) reveal the almost steady evolutions except for some small oscillations as time close to 1 and 2 . With adding a 5\% noise to the initial solution, the simulated evolutions exhibit obviously strong oscillations and instability.

\section{Dynamics of multi-soliton solutions of Eq. (1) via numerical simulations}

In this part, we will give some explicit solutions of Eq. (1) via transformations (9) and (20), and we will compare the numerical evolution results of Eq. (1) with its corresponding two higher-order Toda lattice Eqs. (5) and (6) by use of numerical simulations. Substituting a trivial solution $u_{n}=v_{n}=1$ into (2) and (3), we can give one solution of the Lax pair (2) and 

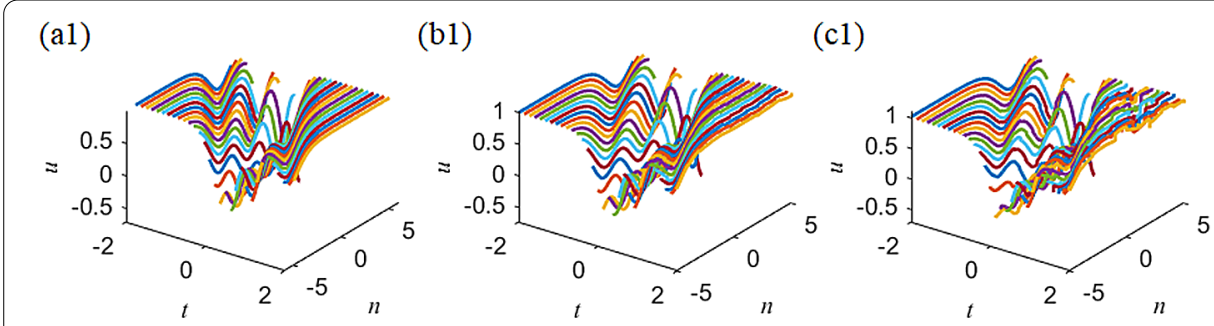

(a2)

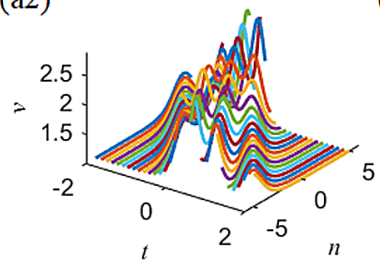

(b2)

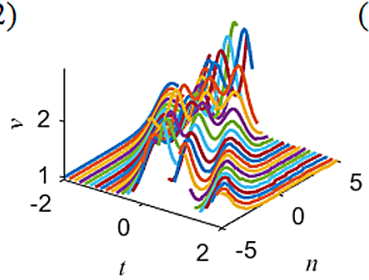

(c2)

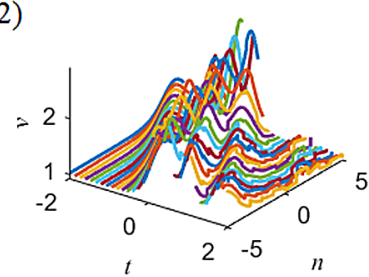

Figure 12 Collisions of four-soliton solutions $\widetilde{u}_{n}$ and $\widetilde{v}_{n}$ in (50) with $N=2$ via the parameters $r_{1}=\frac{1}{2}, r_{2}=-\frac{1}{2}$, $r_{3}=1, r_{4}=-1, \lambda_{1}=-3, \lambda_{2}=-\frac{5}{2}, \lambda_{3}=-2, \lambda_{4}=-\frac{3}{2}$. (a) Exact solution; (b) Time evolution using exact solution as an initial condition; (c) Time evolution using exact solution perturbed by a $2 \%$ noise as the initial condition

(3) with $\lambda=\lambda_{i}(i=1,2, \ldots, 2 N)$ as follows:

$$
\varphi=\left(\begin{array}{c}
\tau_{1}^{n} e^{\rho_{1} t} \\
\tau_{1}^{n+1} e^{\rho_{1} t}
\end{array}\right), \quad \psi=\left(\begin{array}{c}
\tau_{2}^{n} e^{\rho_{2} t} \\
\tau_{2}^{n+1} e^{\rho_{2} t}
\end{array}\right),
$$

where

$$
\begin{array}{ll}
\tau_{1}=\frac{\lambda}{2}-\frac{1}{2}+\frac{\sqrt{\lambda^{2}-2 \lambda-3}}{2}, & \tau_{2}=\frac{\lambda}{2}-\frac{1}{2}-\frac{\sqrt{\lambda^{2}-2 \lambda-3}}{2}, \\
\rho_{1}=\frac{3}{2}-\frac{\lambda}{2}+\frac{\sqrt{\lambda^{2}-2 \lambda-3}}{2}, & \rho_{2}=\frac{3}{2}-\frac{\lambda}{2}-\frac{\sqrt{\lambda^{2}-2 \lambda-3}}{2} .
\end{array}
$$

Therefore, the explicit solutions of Eq. (1) are also given as follows:

$$
\widetilde{u}_{n}=1+d_{n}^{(N-1)}-d_{n+1}^{(N-1)}, \quad \widetilde{v}_{n}=\frac{1+c_{n}^{(N-1)}}{1-b_{n}^{(N-1)}}
$$

where $b_{n}^{(N-1)}, c_{n}^{(N-1)}, d_{n}^{(N-1)}$ are the same as those in Sects. 3 and 4, so we omit their expressions here. The soliton interactions and structures of Eq. (1) are similar to those of Eqs. (5) and (6). Next, we only implement the numerical simulations to display the dynamical behaviors of the previous dark multi-solitons of Eq. (1). Figures 13-16 exhibit the exact one-, two-, three-, and four-soliton solutions (52) of Eq. (1), time evolutions of using exact onesoliton solution (52) and those perturbed by a relatively big noise 20\%. Figures 13-16(a1), (b1)-(a2), (b2) show that the profiles of the time evolution of the one-soliton solution (52) without a noise almost agree with the ones of the corresponding exact one-soliton solution (52) in a larger time $t \in(-2,2)$ or $t \in(-4,4)$. It is clearly seen that our numerical solutions exactly reproduce the analytical solutions; in other words, these solutions have the stable evolutions without a noise, which also shows the accuracy of our numerical scheme. We further numerically study the stability of these solutions by perturbing the above initial conditions (i.e., a relatively big noise $(20 \%)$ is added to both the initial exact solutions 


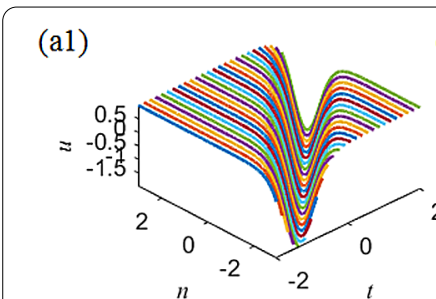

(a2)

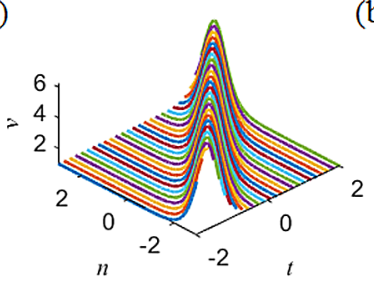

(b1)

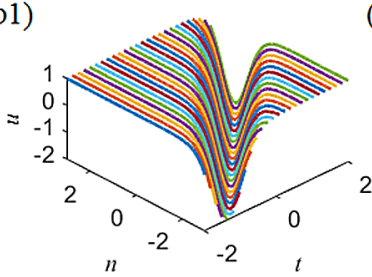

(b2)

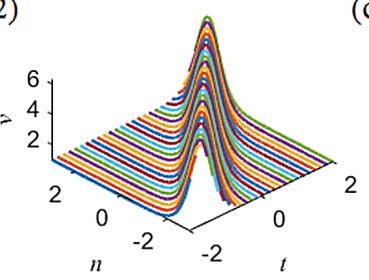

(c1)

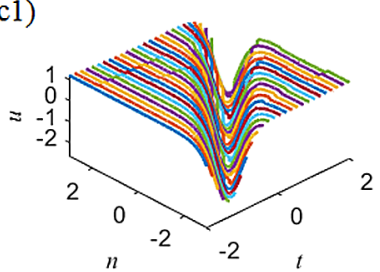

(c2)

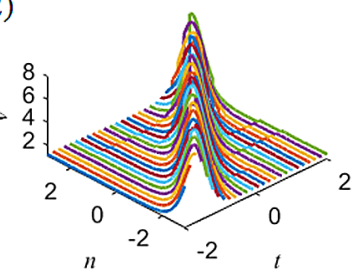

Figure 13 One-soliton solution (52) with the parameters $r_{1}=-1, r_{2}=1, \lambda_{1}=-1, \lambda_{2}=-4$ when $N=1$. (a) Exact solution; (b) Time evolution using exact solution (52) as an initial condition; (c) Time evolution using exact solution (52) perturbed by a $20 \%$ noise as the initial condition

(a1)

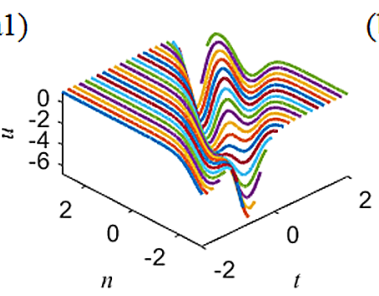

(b1)

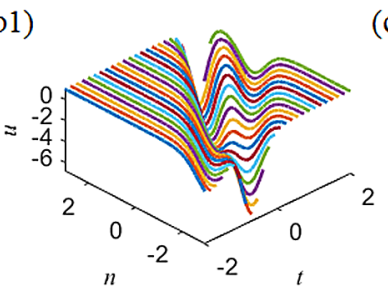

(c1)

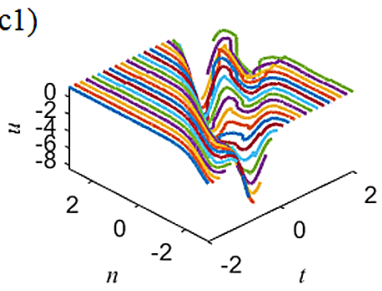

(a2)

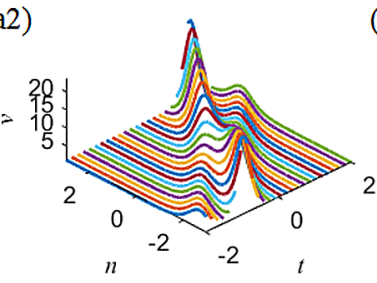

(b2)

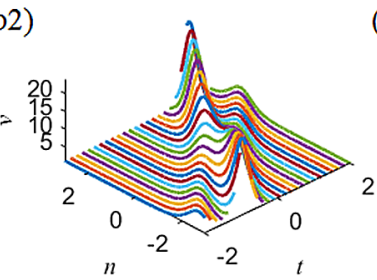

(c2)

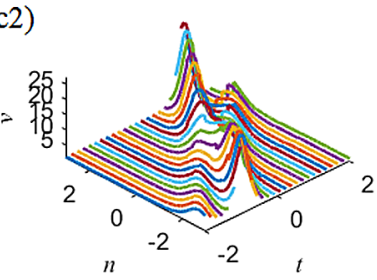

Figure 14 Two-soliton solution (52) with the parameters $r_{1}=-1, r_{2}=1, \lambda_{1}=-4, \lambda_{2}=-9$ when $N=1$. (a) Exact solution; (b) Time evolution using exact solution (52) as an initial condition; (c) Time evolution using exact solution (52) perturbed by a $20 \%$ noise as the initial condition

$u_{n}$ and $v_{n}$ components), the numerical results in Figs. 13-16(c1)-(c2) show that they can evolve as before, that is to say, these soliton solutions (52) of Eq. (1) are robust against a relatively big noise (20\%). In fact, when adding a $30 \%$ noise to their initial solutions, the simulated evolutions of these soliton solutions exhibit very small oscillations.

Compared with the numerical evolution results of Eqs. (1), (5), and (6), we can see that the multi-soliton solutions of Eqs. (1), (5), and (6) can still keep stable evolutions under a small perturbation (a small noise 2\%) except for some small oscillations for Eq. (6). However, the simulated evolutions exhibit obviously strong oscillations and instability for the multi-soliton solutions of Eq. (6) with adding a 5\% noise to their initial solutions, while the multi-soliton solutions of Eqs. (1) and (5) have almost stable evolutions under a small noise (5\%). When adding a $20 \%$ big noise to their initial solutions, we find that the simulated evolutions exhibit obviously strong oscillations and instability for the multi-soliton 


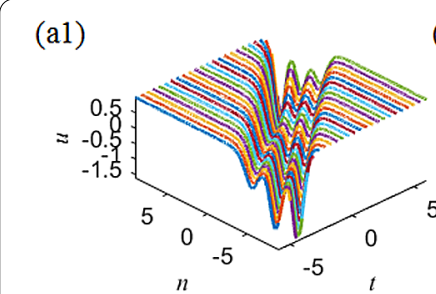

(a2)

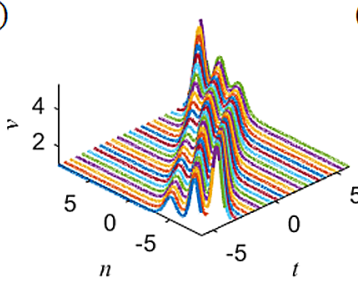

(b1)

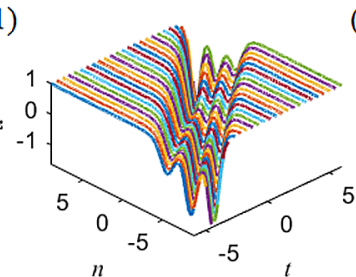

(b2)

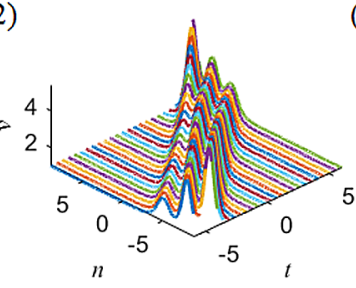

(c1)

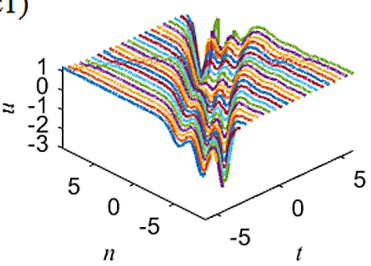

(c2)

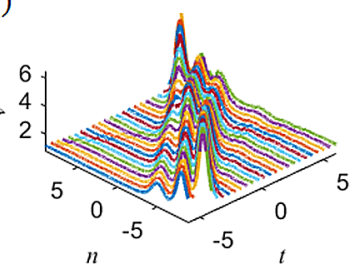

Figure 15 Three-soliton solution (52) with the parameters $r_{1}=1, r_{2}=-1, r_{3}=\frac{1}{2}, r_{2}=-\frac{1}{2}, \lambda_{1}=-4, \lambda_{2}=-3$, $\lambda_{3}=-2, \lambda_{4}=-1$ when $N=2$. (a) Exact solution; (b) Time evolution using exact solution (52) as an initial condition; (c) Time evolution using exact solution (52) perturbed by a $20 \%$ noise as the initial condition

(a1)

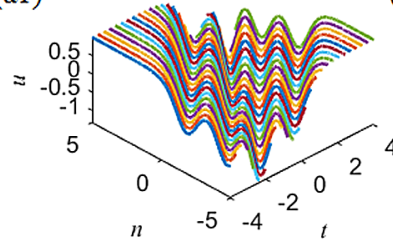

(a2)

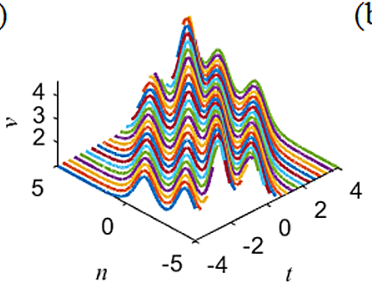

(b1)

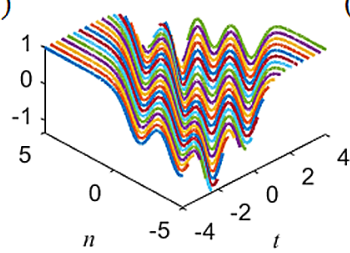

(c1)

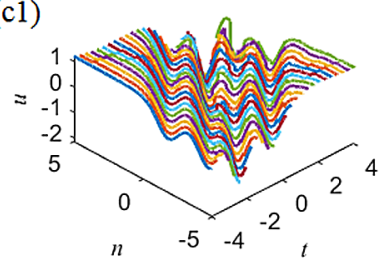

(b2)

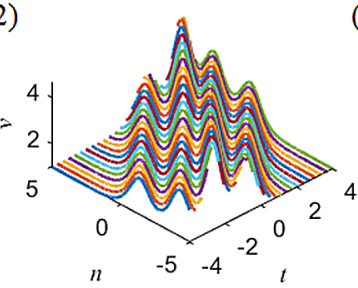

(c2)

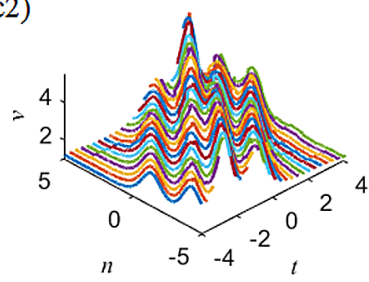

Figure 16 Four-soliton solution (52) with the parameters $r_{1}=1, r_{2}=-1, r_{3}=\frac{1}{2}, r_{2}=-\frac{1}{2}, \lambda_{1}=-4, \lambda_{2}=-3$, $\lambda_{3}=-2.5, \lambda_{4}=-2$ when $N=2$. (a) Exact solution; (b) Time evolution using exact solution (52) as an initial condition; (c) Time evolution using exact solution (52) perturbed by a $20 \%$ noise as the initial condition

solutions of Eqs. (5) and (6), while the multi-soliton solutions of Eq. (1) still have stable evolutions; that is to say, the same small noise has different effect on the evolutions of the dark multi-soliton solutions for three equations. We can see that the multi-soliton solutions of higher-order equations in the same hierarchy are weaker against a small noise than the lower-order equations. We infer that the higher-order nonlinear terms of the higherorder equations (5) and (6) may be the possible reason for the instability of the simulated evolutions.

\section{Discrete generalized ( $n, N-n)$-fold Darboux transformation of Eqs. (1), (5), and (6)}

In Refs. [38, 39], we have proposed the discrete generalized $(n, N-n)$-fold DTs to solve two discrete integrable NLEs. In this section, we will extend this idea to discrete Eqs. (1), 
(5), and (6) and propose their discrete generalized $(n, N-n)$-fold DTs for seeking some new rational solutions. Due to the similar steps for solving three equations, we here only give some rational form solutions of Eq. (1) via our proposed generalized $(n, N-n)$-fold DT technique. For the $N$-fold DT with $2 N$ distinct eigenvalues in Sect. 2, we can give the $2 N$-soliton solutions of Eqs. (1), (5), and (6). However, to derive some new rational solutions of Eqs. (1), (5), and (6), this cannot be done for the $N$-fold DT with $2 N$ distinct eigenvalues, but we can change the number $n$ of the eigenvalues $\lambda$ to construct a new generalized $(n, N-n)$-fold DT to achieve our aim. Next, let us modify the $N$-fold DT in Sect. 2 in order to express some rational form solutions in terms of the determinants of Eqs. (1), (5), and (6). Here we use $n$ distinct eigenvalues $\lambda_{i}(i=1,2, \ldots, n), 1 \leq n \leq 2 N$ and their corresponding highest order $m_{i}\left(m_{i}=0,1,2, \ldots\right)$ derivatives of the solutions of the Lax pair, and we demand that these nonnegative integers $n, m_{i}$ are required to satisfy $2 N=n+\sum_{i=1}^{n} m_{i}$, where $N$ is the same as the one in the Darboux matrix $T$ in Sect. 2. If $1 \leq n \leq 2 N$, then a discrete version of generalized ( $n, N-n)$-fold DT of Eqs. (1), (5), and (6) is described by the following theorem.

Theorem 2 Let $\varphi\left(\lambda_{i}\right)=\left(\phi_{i}\left(\lambda_{i}\right), \psi_{i}\left(\lambda_{i}\right)\right)^{T} \equiv\left(\phi_{i}, \psi_{i}\right)^{T}(i=1,2, \ldots, n)$ be column vector solutions of the spectral problem (2), (3), (7), and (8) for the spectral parameters $\lambda_{i}(i=1,2, \ldots, n)$ with the initial solution $u_{n}, v_{n}$ of Eqs. (1), (5), and (6). Then the generalized perturbation $(n, N-n)$-fold DT of Eq. (1) given by

$$
\widetilde{u}_{n}=u_{n}+d_{n}^{(N-1)}-d_{n+1}^{(N-1)}, \quad \widetilde{v}_{n}=\frac{v_{n}+c_{n}^{(N-1)}}{1-b_{n}^{(N-1)}}
$$

is also a solution of Eqs. (1), (5), and (6), in which

$$
b_{n}^{(N-1)}=\frac{\Delta b_{n}^{(N-1)}}{\Delta_{1}}, \quad c_{n}^{(N-1)}=\frac{\Delta c_{n}^{(N-1)}}{\Delta_{2}}, \quad d_{n}^{(N-1)}=\frac{\Delta d_{n}^{(N-1)}}{\Delta_{2}},
$$

where $\Delta_{1}=\left(\Delta_{1}^{(1)}, \Delta_{1}^{(2)}, \ldots, \Delta_{1}^{(n)}\right)^{T}, \Delta_{2}=\left(\Delta_{2}^{(1)}, \Delta_{2}^{(2)}, \ldots, \Delta_{2}^{(n)}\right)^{T}, \Delta_{1}^{(i)}=\left(\Delta_{1, j, s}^{(i)}\right)_{\left(m_{i}+1\right) \times 2 N}, \Delta_{2}^{(i)}=$ $\left(\Delta_{2, j, s}^{(i)}\right)_{\left(m_{i}+1\right) \times 2 N}$, in which $\Delta_{1}^{(i)}$ and $\Delta_{2}^{(i)}\left(1 \leq j \leq m_{i}+1,1 \leq s \leq 2 N, i=1,2, \ldots, n\right)$ being given by the following formulae:

$$
\begin{gathered}
\Delta_{1, j, s}^{(i)}=\left\{\begin{array}{c}
\sum_{k=0}^{j-1} C_{N-s}^{k} \lambda_{i}^{N-s-k} \phi_{i}^{(j-1-k)} \\
\text { for } 1 \leq j \leq m_{i}+1,1 \leq s \leq N, \\
\sum_{k=0}^{j-1} C_{2 N-s}^{k} \lambda_{i}^{s-k-1} \psi_{i}^{j-k}-\sum_{k=0}^{j-1} C_{2 N-s+1}^{k} \lambda_{i}^{2 N-s-k+1} \phi_{i}^{j-k-1} \\
\text { for } 1 \leq j \leq m_{i}+1, N+1 \leq s \leq 2 N,
\end{array}\right. \\
\Delta_{2, j, s}^{(i)}= \begin{cases}\sum_{k=0}^{j-1} C_{N-s}^{k} \lambda_{i}^{N-s-k} \phi_{i}^{(j-1-k)} & \text { for } 1 \leq j \leq m_{i}+1,1 \leq s \leq N, \\
\sum_{k=0}^{j-1} C_{2 N-s}^{k} \lambda_{i}^{2 N-s-k} \psi_{i}^{j-k-1} & \text { for } 1 \leq j \leq m_{i}+1, N+1 \leq s \leq 2 N,\end{cases}
\end{gathered}
$$

where $\Delta b_{n}^{(N-1)}$ is given from the determinant $\Delta_{1}$ by substituting the column vector $\left(b_{1}^{(1)}, b_{2}^{(1)}, \ldots, b_{m_{1}+1}^{(1)}, \ldots, b_{1}^{(i)}, b_{2}^{(i)}, \ldots, b_{m_{i}+1}^{(i)}, \ldots, b_{1}^{(n)}, b_{2}^{(n)}, \ldots, b_{m_{n}+1}^{(n)}\right)$ in which $b_{j}^{(i)}=-\sum_{k=0}^{j-1} C_{N}^{k} \times$ $\lambda_{i}^{N-k} \phi_{i}^{(j-k-1)}\left(1 \leq j \leq m_{i}+1, i=1,2, \ldots, n\right)$ for its $(N+1)$ th column, while $\Delta c_{n}^{(N-1)}$ and $\Delta d_{n}^{(N-1)}$ are obtained from the determinant $\Delta_{2}$ by respectively replacing the first and $(N+$ 1) th columns with the column vector $\left(c_{1}^{(1)}, c_{2}^{(1)}, \ldots, c_{m_{1}+1}^{(1)}, \ldots, c_{1}^{(i)}, c_{2}^{(i)}, \ldots, c_{m_{i}+1}^{(i)}, \ldots, c_{1}^{(n)}, c_{2}^{(n)}, \ldots\right.$, 
$\left.c_{m_{n}+1}^{(n)}\right)$ in which $c_{j}^{(i)}=-\sum_{k=0}^{j-1} C_{N}^{k} \lambda_{i}^{N-k} \psi_{i}^{(j-k-1)}\left(1 \leq j \leq m_{i}+1, i=1,2, \ldots, n\right)$. Moreover, the terms $\Delta d_{n+1}^{(N)}$ can be derived from $\Delta d_{n}^{(N)}$ by replacing $n$ with $n+1$.

For the discrete generalized $(n, N-n)$-fold DT in Theorem 2, the number $n$ is the number of the distinct spectral parameters and $2 N-n=\sum_{i=1}^{n} m_{i}$ is the sum of the orders of the highest derivative of the Darboux matrix $T_{n}$ in system (13) or the vector eigenfunction $\varphi_{n}$. When $n=2 N$ and $m_{i}=0$, Theorem 2 reduces to the usual discrete $N$-fold DT in Theorem 1; when $n=1$ and $m_{1}=2 N-1$, Theorem 2 reduces to the discrete generalized $(1, N-1)$-fold DT, which will be used to derive higher-order rational solutions of Eqs. (1), (5), and (6) from the initial seed solution in the next section. When $n \neq 1$ or $2 N$, we can derive some other new DTs which will not be discussed here.

\section{Rational solutions of Eq. (1) from non-vanishing background}

In this section, we will use the discrete generalized $(1, N-1)$-fold DT (i.e., discrete generalized $(n, N-n)$-fold DT with $n=1)$ with the single eigenvalue to investigate some new rational solutions of Eq. (1) from the initial non-vanishing background. According to the similar steps, we can also give some discrete rational solutions for Eqs. (5) and (6) which are not discussed here. Next we only take Eq. (1) as an example. Choosing the seed solutions $u_{n}=v_{n}=1$, we can give one combined solution of the Lax pair (2) and (3) from (51) as follows:

$$
\varphi=C_{1}\left(\begin{array}{c}
\tau_{1}^{n} e^{\rho_{1} t} \\
\tau_{1}^{n+1} e^{\rho_{1} t}
\end{array}\right)+C_{2}\left(\begin{array}{c}
\tau_{2}^{n} e^{\rho_{2} t} \\
\tau_{2}^{n+1} e^{\rho_{2} t}
\end{array}\right),
$$

with

$$
\begin{aligned}
\rho_{1} & =\frac{3}{2}-\frac{1}{2} \lambda+\frac{1}{2} \sqrt{\lambda^{2}-2 \lambda-3}, & \rho_{2} & =\frac{3}{2}-\frac{1}{2} \lambda-\frac{1}{2} \sqrt{\lambda^{2}-2 \lambda-3}, \\
\tau_{1} & =\frac{1}{2} \lambda-\frac{1}{2}+\frac{1}{2} \sqrt{\lambda^{2}-2 \lambda-3}, & \tau_{2} & =\frac{1}{2} \lambda-\frac{1}{2}-\frac{1}{2} \sqrt{\lambda^{2}-2 \lambda-3},
\end{aligned}
$$

where $C_{1}$ and $C_{2}$ are arbitrary real constants and $\varepsilon$ is a small parameter. In what follows, we fix the spectral parameter $\lambda=\lambda_{1}+\varepsilon^{2}$ with $\lambda_{1}=3$ in Eq. (55), then expand the vector function $\varphi$ in Eq. (55) as two Taylor series at $\varepsilon=0$. Here we choose some special $C_{1}, C_{2}$ (e.g., $C_{1}=\varepsilon, C_{2}=\varepsilon$ ) and then obtain

$$
\varphi\left(\varepsilon^{2}\right)=\varphi^{(0)}+\varphi^{(1)} \varepsilon^{2}+\varphi^{(2)} \varepsilon^{4}+\varphi^{(3)} \varepsilon^{6}+\cdots,
$$

where

$$
\begin{aligned}
& \varphi^{(0)}=\left(\begin{array}{l}
\phi^{(0)} \\
\psi^{(0)}
\end{array}\right)=\left(\begin{array}{l}
2 \\
2
\end{array}\right), \\
& \varphi^{(1)}=\left(\begin{array}{l}
\phi^{(1)} \\
\psi^{(1)}
\end{array}\right)=\left(\begin{array}{c}
n^{2}+2 n t+t^{2}-t \\
n^{2}+(2 t+2) n+t^{2}+t+1
\end{array}\right),
\end{aligned}
$$




$$
\begin{aligned}
\varphi^{(2)} & =\left(\begin{array}{l}
\phi^{(2)} \\
\psi^{(2)}
\end{array}\right) \\
& =\left(\begin{array}{c}
\frac{1}{12} n^{4}+\frac{1}{3} t n^{3}+\frac{1}{2}\left(t^{2}-t-\frac{1}{6}\right) n^{2}+\left(\frac{1}{3} t^{3}-t^{2}+\frac{1}{6} t\right) n+\frac{1}{12} t^{4}-\frac{1}{2} t^{3}+\frac{1}{2} t^{2} \\
\frac{1}{12} n^{4}+\frac{1}{3}(t+1) n^{3}+\frac{1}{12}\left(6 t^{2}+6 t+5\right) n^{2}+\frac{1}{6}\left(2 t^{3}+t+1\right) n+\frac{1}{12} t^{3}(t-2)
\end{array}\right),
\end{aligned}
$$

and $\left(\phi^{(i)}, \psi^{(i)}\right)^{T}(i=3,4,5, \ldots)$ are omitted here. By applying (53), we can give some new rational form solutions of Eq. (1). Next, we will give some rational form solutions via the foregoing proposed discrete generalized $(1, N-1)$-fold DT with $N=1,2,3$.

(I) When $N=1$, according to Theorem 2 , based on the generalized $(1,0)$-fold DT, the first-order rational solution of Eq. (1) is given as

$$
\widetilde{u}_{n}=u_{n}+d_{n}^{(0)}-d_{n+1}^{(0)}, \quad \widetilde{v}_{n}=\frac{v_{n}+c_{n}^{(0)}}{1-b_{n}^{(0)}},
$$

where

$$
b_{n}^{(0)}=\frac{\Delta b_{n}^{(0)}}{\Delta_{1}}, \quad c_{n}^{(0)}=\frac{\Delta c_{n}^{(0)}}{\Delta_{2}}, \quad d_{n}^{(0)}=\frac{\Delta d_{n}^{(0)}}{\Delta_{2}}
$$

with

$$
\begin{aligned}
& \Delta_{1}=\left|\begin{array}{cc}
\phi^{(0)} & -\lambda \phi^{(0)}+\psi^{(0)} \\
\phi^{(1)} & -\lambda \phi^{(1)}+\psi^{(1)}-\phi^{(0)}
\end{array}\right|, \quad \Delta b_{n}^{(0)}=\left|\begin{array}{cc}
\phi^{(0)} & -\lambda \phi^{(0)} \\
\phi^{(1)} & -\lambda \phi^{(1)}-\phi^{(0)}
\end{array}\right|, \\
& \Delta_{2}=\left|\begin{array}{ll}
\phi^{(0)} & \psi^{(0)} \\
\phi^{(1)} & \psi^{(1)}
\end{array}\right|, \quad \Delta c_{n}^{(0)}=\left|\begin{array}{cc}
-\lambda \psi^{(0)} & \psi^{(0)} \\
-\lambda \psi^{(1)}-\psi^{(0)} & \psi^{(1)}
\end{array}\right|, \\
& \Delta d_{n}^{(0)}=\left|\begin{array}{cc}
\phi^{(0)} & -\lambda \psi^{(0)} \\
\phi^{(1)} & -\lambda \psi^{(1)}-\psi^{(0)}
\end{array}\right| .
\end{aligned}
$$

The simplification form of solution (60) is listed as follows:

$$
\widetilde{u}_{1}=\frac{4 n^{2}+8(t+1) n+4 t^{2}+8 t-1}{(2 n+2 t+3)(2 n+2 t+1)}, \quad \widetilde{v}_{1}=\frac{(2 n+2 t+3)(2 n+2 t-1)}{(2 n+2 t+1)^{2}}
$$

from which we can see that $\widetilde{u}_{1}$ possesses singularity at two straight lines $2 n+2 t+3=0$ and $2 n+2 t+1=0$, while $\widetilde{v}_{1}$ possesses singularity at the straight line $2 n+2 t+1=0$.

(II) When $N=2$, according to Theorem 2, based on the generalized $(1,1)$-fold DT, the second-order rational solution of Eq. (1) is given as

$$
\widetilde{u}_{n}=u_{n}+d_{n}^{(1)}-d_{n+1}^{(1)}, \quad \widetilde{v}_{n}=\frac{v_{n}+c_{n}^{(1)}}{1-b_{n}^{(1)}}
$$

where

$$
b_{n}^{(1)}=\frac{\Delta b_{n}^{(1)}}{\Delta_{1}}, \quad c_{n}^{(1)}=\frac{\Delta c_{n}^{(1)}}{\Delta_{2}}, \quad d_{n}^{(1)}=\frac{\Delta d_{n}^{(1)}}{\Delta_{2}}
$$


with

$$
\begin{aligned}
& \Delta_{1}=\left|\begin{array}{cccc}
\lambda \phi^{(0)} & \phi^{(0)} & -\lambda^{2} \phi^{(0)}+\lambda \psi^{(0)} & \psi^{(0)} \\
\lambda \phi^{(1)}+\phi^{(0)} & \phi^{(1)} & -\lambda^{2} \phi^{(1)}-2 \lambda \phi^{(0)}+\lambda \psi^{(1)}+\psi^{(0)} & \psi^{(1)} \\
\lambda \phi^{(2)}+\phi^{(1)} & \phi^{(2)} & -\lambda^{2} \phi^{(2)}-2 \lambda \phi^{(1)}-\phi^{(0)}+\lambda \psi^{(2)}+\psi^{(1)} & \psi^{(2)} \\
\lambda \phi^{(3)}+\phi^{(2)} & \phi^{(3)} & -\lambda^{2} \phi^{(3)}-2 \lambda \phi^{(2)}-\phi^{(1)}+\lambda \psi^{(3)}+\psi^{(2)} & \psi^{(3)}
\end{array}\right| \\
& \Delta b_{n}^{(1)}=\left|\begin{array}{cccc}
\lambda \phi^{(0)} & \phi^{(0)} & -\lambda^{2} \phi^{(0)} & \psi^{(0)} \\
\lambda \phi^{(1)}+\phi^{(0)} & \phi^{(1)} & -\lambda^{2} \phi^{(1)}-2 \lambda \phi^{(0)} & \psi^{(1)} \\
\lambda \phi^{(2)}+\phi^{(1)} & \phi^{(2)} & -\lambda^{2} \phi^{(2)}-2 \lambda \phi^{(1)}-\phi^{(0)} & \psi^{(2)} \\
\lambda \phi^{(3)}+\phi^{(2)} & \phi^{(3)} & -\lambda^{2} \phi^{(3)}-2 \lambda \phi^{(2)}-\phi^{(1)} & \psi^{(3)}
\end{array}\right| \\
& \Delta_{2}=\left|\begin{array}{cccc}
\lambda \phi^{(0)} & \phi^{(0)} & \lambda \psi^{(0)} & \psi^{(0)} \\
\lambda \phi^{(1)}+\phi^{(0)} & \phi^{(1)} & \lambda \psi^{(1)}+\psi^{(0)} & \psi^{(1)} \\
\lambda \phi^{(2)}+\phi^{(1)} & \phi^{(2)} & \lambda \psi^{(2)}+\psi^{(1)} & \psi^{(2)} \\
\lambda \phi^{(3)}+\phi^{(2)} & \phi^{(3)} & \lambda \psi^{(3)}+\psi^{(2)} & \psi^{(3)}
\end{array}\right| . \\
& \Delta c_{n}^{(1)}=\left|\begin{array}{cccc}
-\lambda^{2} \psi^{(1)}-2 \lambda \psi^{(0)} & \phi^{(1)} & \lambda \psi^{(1)}+\psi^{(0)} & \psi^{(1)} \\
-\lambda^{2} \psi^{(2)}-2 \lambda \psi^{(1)}-\psi^{(0)} & \phi^{(2)} & \lambda \psi^{(2)}+\psi^{(1)} & \psi^{(2)} \\
-\lambda^{2} \psi^{(3)}-2 \lambda \psi^{(2)}-\psi^{(1)} & \phi^{(3)} & \lambda \psi^{(3)}+\psi^{(2)} & \psi^{(3)}
\end{array}\right| \\
& \Delta d_{n}^{(1)}=\left|\begin{array}{cccc}
\lambda \phi^{(0)} & \phi^{(0)} & -\lambda^{2} \psi^{(0)} & \psi^{(0)} \\
\lambda \phi^{(1)}+\phi^{(0)} & \phi^{(1)} & -\lambda^{2} \psi^{(1)}-2 \lambda \psi^{(0)} & \psi^{(1)} \\
\lambda \phi^{(2)}+\phi^{(1)} & \phi^{(2)} & -\lambda^{2} \psi^{(2)}-2 \lambda \psi^{(1)}-\psi^{(0)} & \psi^{(2)} \\
\lambda \phi^{(3)}+\phi^{(2)} & \phi^{(3)} & -\lambda^{2} \psi^{(3)}-2 \lambda \psi^{(2)}-\psi^{(1)} & \psi^{(3)}
\end{array}\right|
\end{aligned}
$$

The simplification form of solution (64) is listed as follows:

$$
\tilde{u}_{2}=\frac{\Omega_{1}}{\Omega_{2}}, \quad \widetilde{v}_{2}=\frac{\Omega_{3}}{\Omega_{4}},
$$

where

$$
\begin{aligned}
\Omega_{1}= & -813,600 n t^{4}+17,412 n^{3}-89,760 n^{5}+1890 n-223,920 t^{6}-1,230,192 n t^{5} \\
& -3,343,840 n^{3} t^{3}+783,360 t^{7} n^{2}-111,472 n^{6}+14,336 n^{10}+3072 n^{11} t \\
& -3,055,680 n^{4} t^{3}-24,480 t^{8}+120,000 n^{5} t^{3}+3,225,600 t^{4} n^{5}+11,061 n^{2} \\
& +16,896 t^{1} 0 n^{2}+3072 t^{1} 1+131,840 t^{9} n+256 n^{12}-135,360 t^{7}+599,040 t^{8} n^{2} \\
& +3072 n^{11}+28,350 t^{2}+168,960 t^{9} n^{2}+1,013,760 t^{4} n^{7}-2,791,440 n^{2} t^{4}+256 t^{1} 2 \\
& +506,880 t^{8} n^{3}+104,256 n^{7} t-501,360 n^{4} t-778,800 t n^{5}-390,144 t^{6} n^{2} \\
& +3072 t^{1} 1 n+506,880 t^{3} n^{8}+2,849,280 t^{6} n^{4}-148,320 n t^{3}+8100 t+184,320 t^{8} n \\
& +21,600 t^{3}-36,528 t n^{3}+3,451,392 t^{5} n^{5}+56,320 t^{9} n^{3}-153,792 t^{7} n-192,240 t^{5} \\
& -482,112 t^{5} n^{3}+112,860 n t^{2}+2,257,920 t^{3} n^{6}+126,720 n^{8} t^{4}-3,490,560 n^{3} t^{4} \\
& -1,566,720 n^{5} t^{2}+18,528 n^{8}+19,200 t^{9}+211,968 n^{6} t^{2}+30,720 n^{9}+33,792 t^{1} 0 n \\
& +1,612,800 t^{7} n^{3}+33,792 n^{10} t+126,720 t^{8} n^{4}+1,674,240 t^{3} n^{7}+1,419,264 t^{6} n^{5}
\end{aligned}
$$




$$
\begin{aligned}
& -48,384 n^{7}+13,056 t^{1} 0+633,600 t^{2} n^{8}+264,960 n^{8} t+1,013,760 t^{2} n^{7} \\
& -227,520 t^{4} n^{4}+3,064,320 t^{5} n^{4}-98,514 n^{2} t^{2}+1,419,264 t^{5} n^{6}-1,372,320 n^{2} t^{3} \\
& -68,175 t^{4}+142,080 t n^{9}+202,752 t^{7} n^{5}+56,320 n^{9} t^{3}+16,896 n^{10} t^{2} \\
& -2,226,000 n^{4} t^{2}+115,632 n^{2} t-2,349,504 n^{2} t^{5}+236,544 t^{6} n^{6}+1,935,360 t^{6} n^{3} \\
& -17,759 n^{4}+2,903,040 t^{4} n^{6}-1,162,560 n^{3} t^{2}-431,424 t n^{6}-866,304 n t^{6} \\
& +202,752 n^{7} t^{5}+168,960 t^{2} n^{9}+1,013,760 t^{7} n^{4}+55,620 n t, \\
& \Omega_{2}=\left(90+471 n+520 n^{4}+949 n^{2}+16 n^{6}+480 t^{4}+320 n^{3} t^{3}+16 t^{6}+144 t^{5}+780 t^{3}\right. \\
& +96 t n^{5}+1644 n t+2040 t n^{3}+1960 n t^{3}+2520 n t^{2}+3000 n^{2} t^{2}+2700 n^{2} t \\
& +1440 n^{2} t^{3}+1440 n^{3} t^{2}+720 n t^{4}+96 n t^{5}+240 n^{2} t^{4}+360 t+144 n^{5}+240 n^{4} t^{2} \\
& \left.+675 t^{2}+960 n^{3}+720 n^{4} t\right)\left(-3 n-60 t^{3}-45 t^{2}+48 t^{5}+16 t^{6}-36 n t+120 t n^{3}\right. \\
& +40 n t^{3}-120 n t^{2}+120 n^{2} t^{2}-60 n^{2} t-11 n^{2}+40 n^{4}+480 n^{2} t^{3}+480 n^{3} t^{2}+48 n^{5} \\
& \left.+16 n^{6}+240 n t^{4}+96 n t^{5}+240 n^{2} t^{4}+320 n^{3} t^{3}+240 n^{4} t+240 n^{4} t^{2}+96 t n^{5}\right), \\
& \Omega_{3}=\left(90+520 n^{4}+3000 n^{2} t^{2}+2520 n t^{2}+1960 n t^{3}+2040 t n^{3}+1644 n t+471 n\right. \\
& +2700 n^{2} t+240 n^{4} t^{2}+144 n^{5}+720 n^{4} t+320 n^{3} t^{3}+949 n^{2}+1440 n^{3} t^{2} \\
& +1440 n^{2} t^{3}+96 n t^{5}+720 n t^{4}+240 n^{2} t^{4}+360 t+16 n^{6}+960 n^{3}+96 t n^{5}+780 t^{3} \\
& \left.+480 t^{4}+675 t^{2}+144 t^{5}+16 t^{6}\right)\left(3 n+60 t^{3}-45 t^{2}-48 t^{5}+16 t^{6}-36 n t+120 t n^{3}\right. \\
& +40 n t^{3}+120 n t^{2}+120 n^{2} t^{2}+60 n^{2} t-11 n^{2}+40 n^{4}-480 n^{2} t^{3}-480 n^{3} t^{2}-48 n^{5} \\
& \left.+16 n^{6}-240 n t^{4}+96 n t^{5}+240 n^{2} t^{4}+320 n^{3} t^{3}-240 n^{4} t+240 n^{4} t^{2}+96 t n^{5}\right), \\
& \Omega_{4}=\left(-3 n-60 t^{3}-45 t^{2}+48 t^{5}+16 t^{6}-36 n t+120 t n^{3}+40 n t^{3}-120 n t^{2}+120 n^{2} t^{2}\right. \\
& -60 n^{2} t-11 n^{2}+40 n^{4}+480 n^{2} t^{3}+480 n^{3} t^{2}+48 n^{5}+16 n^{6}+240 n t^{4}+96 n t^{5} \\
& \left.+240 n^{2} t^{4}+320 n^{3} t^{3}+240 n^{4} t+240 n^{4} t^{2}+96 t n^{5}\right)^{2} \text {. }
\end{aligned}
$$

From $(65)$ we can see that $\tilde{u}_{2}$ possesses singularity at two curves

$$
\begin{aligned}
90 & +471 n+520 n^{4}+949 n^{2}+16 n^{6}+480 t^{4}+320 n^{3} t^{3}+16 t^{6}+144 t^{5}+780 t^{3}+96 t n^{5} \\
& +1644 n t+2040 t n^{3}+1960 n t^{3}+2520 n t^{2}+3000 n^{2} t^{2}+2700 n^{2} t+1440 n^{2} t^{3} \\
& +1440 n^{3} t^{2}+720 n t^{4}+96 n t^{5}+240 n^{2} t^{4}+360 t+144 n^{5}+240 n^{4} t^{2}+675 t^{2} \\
& +960 n^{3}+720 n^{4} t=0
\end{aligned}
$$

and

$$
\begin{aligned}
& -3 n-60 t^{3}-45 t^{2}+48 t^{5}+16 t^{6}-36 n t+120 t n^{3}+40 n t^{3}-120 n t^{2}+120 n^{2} t^{2}-60 n^{2} t \\
& -11 n^{2}+40 n^{4}+480 n^{2} t^{3}+480 n^{3} t^{2}+48 n^{5}+16 n^{6}+240 n t^{4}+96 n t^{5}+240 n^{2} t^{4} \\
& +320 n^{3} t^{3}+240 n^{4} t+240 n^{4} t^{2}+96 t n^{5}=0,
\end{aligned}
$$


while $\widetilde{v}_{1}$ possesses singularity at the curve

$$
\begin{aligned}
-3 n & -60 t^{3}-45 t^{2}+48 t^{5}+16 t^{6}-36 n t+120 t n^{3}+40 n t^{3}-120 n t^{2}+120 n^{2} t^{2}-60 n^{2} t \\
- & 11 n^{2}+40 n^{4}+480 n^{2} t^{3}+480 n^{3} t^{2}+48 n^{5}+16 n^{6}+240 n t^{4}+96 n t^{5}+240 n^{2} t^{4} \\
+ & 320 n^{3} t^{3}+240 n^{4} t+240 n^{4} t^{2}+96 t n^{5}=0 .
\end{aligned}
$$

(III) When $N=3$, according to Theorem 2, based on the generalized $(1,2)$-fold DT, we have the third-order rational solution of Eq. (1) as follows:

$$
\widetilde{u}_{n}=u_{n}+d_{n}^{(2)}-d_{n+1}^{(2)}, \quad \widetilde{v}_{n}=\frac{v_{n}+c_{n}^{(2)}}{1-b_{n}^{(2)}},
$$

where

$$
b_{n}^{(2)}=\frac{\Delta b_{n}^{(2)}}{\Delta_{1}}, \quad c_{n}^{(2)}=\frac{\Delta c_{n}^{(2)}}{\Delta_{2}}, \quad d_{n}^{(2)}=\frac{\Delta d_{n}^{(2)}}{\Delta_{2}}
$$

with

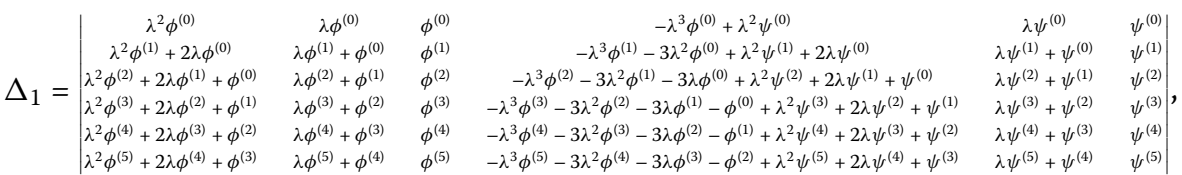

$$
\begin{aligned}
& \Delta_{2}=\left|\begin{array}{cccccc}
\lambda^{2} \phi^{(0)} & \lambda \phi^{(0)} & \phi^{(0)} & \lambda^{2} \psi^{(0)} & \lambda \psi^{(0)} & \psi^{(0)} \\
\lambda^{2} \phi^{(1)}+2 \lambda \phi^{(0)} & \lambda \phi^{(1)}+\phi^{(0)} & \phi^{(1)} & \lambda^{2} \psi^{(1)}+2 \lambda \psi^{(0)} & \lambda \psi^{(1)}+\psi^{(0)} & \psi^{(1)} \\
\lambda^{2} \phi^{(2)}+2 \lambda \phi^{(1)}+\phi^{(0)} & \lambda \phi^{(2)}+\phi^{(1)} & \phi^{(2)} & \lambda^{2} \psi^{(2)}+2 \lambda \psi^{(1)}+\psi^{(0)} & \lambda \psi^{(2)}+\psi^{(1)} & \psi^{(2)} \\
\lambda^{2} \phi^{(3)}+2 \lambda \phi^{(2)}+\phi^{(1)} & \lambda \phi^{(3)}+\phi^{(2)} & \phi^{(3)} & \lambda^{2} \psi^{(3)}+2 \lambda \psi^{(2)}+\psi^{(1)} & \lambda \psi^{(3)}+\psi^{(2)} & \psi^{(3)} \\
\lambda^{2} \phi^{(4)}+2 \lambda \phi^{(3)}+\phi^{(2)} & \lambda \phi^{(4)}+\phi^{(3)} & \phi^{(4)} & \lambda^{2} \psi^{(4)}+2 \lambda \psi^{(3)}+\psi^{(2)} & \lambda \psi^{(4)}+\psi^{(3)} & \psi^{(4)} \\
\lambda^{2} \phi^{(5)}+2 \lambda \phi^{(4)}+\phi^{(3)} & \lambda \phi^{(5)}+\phi^{(4)} & \phi^{(5)} & \lambda^{2} \psi^{(5)}+2 \lambda \psi^{(4)}+\psi^{(3)} & \lambda \psi^{(5)}+\psi^{(4)} & \psi^{(5)}
\end{array}\right|
\end{aligned}
$$

and $\Delta b_{n}^{(2)}$ is produced from $\Delta_{1}$ by replacing its fourth column with $\left(-\lambda^{3} \phi^{(0)},-\lambda^{3} \phi^{(1)}-\right.$ $3 \lambda^{2} \phi^{(0)},-\lambda^{3} \phi^{(2)}-3 \lambda^{2} \phi^{(1)}-3 \lambda \phi^{(0)},-\lambda^{3} \phi^{(3)}-3 \lambda^{2} \phi^{(2)}-3 \lambda \phi^{(1)}-\phi^{(0)},-\lambda^{3} \phi^{(4)}-3 \lambda^{2} \phi^{(3)}-$ $\left.3 \lambda \phi^{(2)}-\phi^{(1)},-\lambda^{3} \phi^{(5)}-3 \lambda^{2} \phi^{(4)}-3 \lambda \phi^{(3)}-\phi^{(2)}\right)^{T}, \Delta c_{n}^{(2)}$ and $\Delta d_{n}^{(2)}$ from $\Delta_{2}$ by replacing its first and fourth columns with $\left(-\lambda^{3} \psi^{(0)},-\lambda^{3} \psi^{(1)}-3 \lambda^{2} \psi^{(0)},-\lambda^{3} \psi^{(2)}-3 \lambda^{2} \psi^{(1)}-3 \lambda \psi^{(0)},-\lambda^{3} \psi^{(3)}-\right.$ $\left.3 \lambda^{2} \psi^{(2)}-3 \lambda \psi^{(1)}-\psi^{(0)},-\lambda^{3} \psi^{(4)}-3 \lambda^{2} \psi^{(3)}-3 \lambda \psi^{(2)}-\psi^{(1)},-\lambda^{3} \psi^{(5)}-3 \lambda^{2} \psi^{(4)}-3 \lambda \psi^{(3)}-\psi^{(2)}\right)^{T}$, respectively. The simplification form of solution (66) is so complicated that it is omitted here.

With symbolic computation Maple, solutions (60) or (62), (63) or (65) and (66) have been verified by substituting them into Eq. (1). Next, we summarize a few mathematical features of the foregoing discrete rational solutions for Eq. (1) and sum up the findings which are shown in Tables 1 and 2. The first column in Table 1 shows the order number of the solutions, while the second and third columns show the highest power of the polynomials involved in each solution. The last column provides the background level of these solutions. We can conclude that the highest powers in the numerator and denominator polynomials are both $2 j(2 j-1)$ for the rational solutions $u_{n}$ and $v_{n}$ of order $j$, and their background levels are 1 . Through analyzing these rational solutions, we can find that they possess singularity. Further research is needed whether or not Eq. (1) has some nonsingular rational solutions. 
Table 1 Main mathematical features of rational solutions $u_{n}$ and $v_{n}$ of order $j$

\begin{tabular}{llll}
\hline$j$ & HPN & HPD & Background \\
\hline 1 & 2 & 2 & 1 \\
2 & 12 & 12 & 1 \\
3 & 30 & 30 & 1 \\
$\ldots$ & $\ldots$ & $\ldots$ & $\ldots$ \\
$j$ & $2 j(2 j-1)$ & $2 j(2 j-1)$ & 1 \\
\hline
\end{tabular}

Here, HPN is the highest power in the numerator, while HPD is the highest power in the denominator.

Table 2 Main mathematical features of rational solutions $u_{n}$ and $v_{n}$ of order $j$

\begin{tabular}{llll}
\hline$j$ & HPN & HPD & Background \\
\hline 1 & 6 & 6 & 1 \\
2 & 20 & 20 & 1 \\
3 & 42 & 42 & 1 \\
$\ldots$ & $\ldots$ & $\ldots$ & $\ldots$ \\
$j$ & $2 j(2 j+1)$ & $2 j(2 j+1)$ & 1 \\
\hline
\end{tabular}

Here, HPN is the highest power in the numerator, while HPD is the highest power in the denominator.

It is particularly worth pointing out that we also derive some new rational solutions from (60), (63), and (66) if we choose $C_{1}=1, C_{2}=-1$ in solution (55) and expand the vector function $\varphi$ in Eq. (55) as two Taylor series at $\varepsilon=0$ when $\lambda=3+\varepsilon^{2}$. We here omit their analytical expressions and only list their properties in Table 2 . According to the same steps for seeking the rational solutions of Eq. (1), we can also give some discrete rational solutions for Eqs. (5) and (6), and their properties are the same as the ones of Eq. (1) in Tables 1 and 2, so we will not discuss them here.

\section{Conclusion}

In this paper, we have studied the discrete bright and dark multi-soliton solutions of two high order Toda lattice equations (5) and (6). The discrete $N$-fold DT (9) and (20) for Eqs. (5) and (6) have been constructed. One-, two-, three-, and four-soliton solutions in terms of determinant for Eqs. (5) and (6) have been derived via the resulting $N$-fold DT, and the propagation and elastic interaction structures of such multi-solitons are shown graphically: Fig. 1 exhibits the overtaking elastic interactions between the bright and dark two-soliton solutions with $N=1$; Fig. 2 shows the evolution structures of the bright and dark one-soliton solutions with $N=1$; Fig. 3 displays the overtaking elastic interactions among the bright and dark four-soliton solutions with $N=2$; Fig. 4 shows the overtaking elastic interactions among the bright and dark three-soliton solutions with $N=2$. Solitonic shapes and amplitudes in Figs. 2-4 do not change before and after the interactions, their interactions are elastic. And it is worth noting that the corresponding $(2 N)$-soliton solutions for both Eqs. (5) and (6) can reduce to the $(2 N-1)$-soliton solutions by choosing spectral parameter $\lambda$. Moreover, the $(2 N)$-soliton and $(2 N-1)$-soliton solutions make up the $N$-soliton solutions for Eqs. (5) and (6). Numerical simulation results in Figs. 5-8 show that the evolutions of the bright and dark soliton solutions of Eq. (5) are stable against a 5\% noise. Compared with Eq. (5), the numerical simulation evolutions in Figs. 9-12 exhibit the almost steady evolutions with a $2 \%$ small noise for Eq. (6). However, with adding the same 5\% noise to Eq. (6) as Eq. (5), the evolutions exhibit obviously strong oscillations and instability. Numerical simulation results in Figs. 13-16 show that the evolutions of the soliton solutions of Eq. (1) are stable against a $20 \%$ noise. When adding a $20 \%$ noise 
to the initial solutions of Eqs. (5) and (6), the soliton evolutions for Eqs. (5) and (6) exhibit obviously strong oscillations and instability, while Eq. (1) still has stable evolutions. In fact, when adding a $30 \%$ noise to the initial solutions for Eq. (1), the simulated evolutions of these soliton solutions only exhibit some small oscillations. We find that the same noise has different effect on the evolutions of the multi-soliton solutions for three different equations in the same hierarchy, and we infer that the possible reason is that the higher-order nonlinear terms in higher-order equations have an important impact on the propagation instability of the solitons relative to their corresponding lower-order equations. Finally, some discrete higher-order rational solutions for Eq. (1) have been derived by applying the discrete generalized $(n, N-n)$-fold DT, and the same process is also applied to Eqs. (5) and (6). Tables 1 and 2 show a few mathematical features of these rational solutions of Eq. (5). The results in this paper might be helpful for understanding the propagation of nonlinear waves in soliton theory.

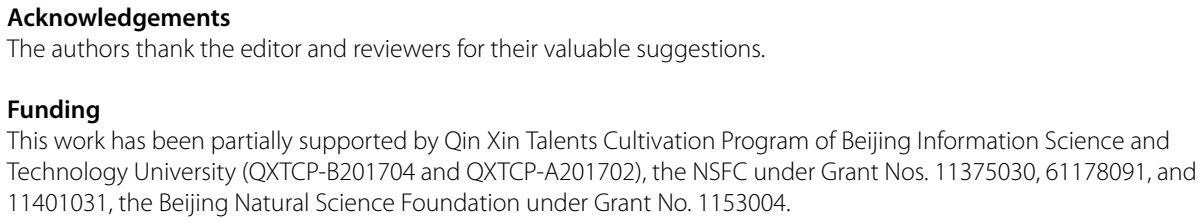

NL performed the theory analysis and carried out the computations. XW participated in the design of the study and the theory analysis, and moreover helped to draft and revise the manuscript. All authors have read and approved the final manuscript.

\section{Publisher's Note}

Springer Nature remains neutral with regard to jurisdictional claims in published maps and institutional affiliations.

Received: 18 January 2018 Accepted: 6 August 2018 Published online: 23 August 2018

\section{References}

1. Ablowitz, M.J., Ladik, J.F.: A nonlinear difference scheme and inverse scattering. Stud. Appl. Math. 55, $213-229$ (1976)

2. Ablowitz, M.J., Ladik, J.F.: Nonlinear differential-difference equations. J. Math. Phys. 16, 598-603 (1975)

3. Ablowitz, M.J.: Nonlinear evolution equations-continuous and discrete. SIAM Rev. 19, 663-684 (1977)

4. Ablowitz, M.J., Clarkson, P.A.: Solitons, Nonlinear Evolution Equations and Inverse Scattering. Cambridge University Press, Cambridge (1991)

5. Ma, W.X., Zhou, Y.: Lump solutions to nonlinear partial differential equations via Hirota bilinear forms. J. Differ. Equ. 264(4), 2633-2659 (2018)

6. Dong, H.H., Zhao, K., Yang, H.Q., Li, Y.Q.: Generalised (2 + 1)-dimensional super MKdV hierarchy for integrable systems in soliton theory. East Asian J. Appl. Math. 5, 256-272 (2015)

7. Li, X.Y., Zhao, Q.L.: A new integrable symplectic map by the binary nonlinearization to the super AKNS system. J. Geom. Phys. 121, 123-137 (2017)

8. Wang, D.S., Wang, X.L.: Long-time asymptotics and the bright $N$-soliton solutions of the Kundu-Eckhaus equation via the Riemann-Hilbert approach. Nonlinear Anal., Real World Appl. 41, 334-361 (2018)

9. Wang, D.S., Wei, X.Q.: Integrability and exact solutions of a two-component Korteweg-de Vries system. Appl. Math. Lett. 51, 60-67 (2016)

10. Wadati, M.: Transformation theories for nonlinear discrete systems. Prog. Theor. Phys. Suppl. 59, 36-63 (1977)

11. Toda, M.: Theory of Nonlinear Lattices. Springer, Berlin (1989)

12. Toda, M.: Vibration of a chain with nonlinear interaction. J. Phys. Soc. Jpn. 22, 431-436 (1967)

13. Hirota, R.: Exact N-soliton solution of a nonlinear lumped network equation. J. Phys. Soc. Jpn. 35, $286-288$ (1973)

14. Kaup, D.J.: Variational solutions for the discrete nonlinear Schrödinger equation. Math. Comput. Simul. 69, 322-333 (2005)

15. Li, X.Y., Zhao, Q.L., Li, Y.X., Dong, H.H.: Binary Bargmann symmetry constraint associated with $3 \times 3$ discrete matrix spectral problem. J. Nonlinear Sci. Appl. 8, 496-506 (2015)

16. Dong, H.H., Chen, T.T., Chen, L.F., Zhang, Y.: A new integrable symplectic map and the Lie point symmetry associated with nonlinear lattice equations. J. Nonlinear Sci. Appl. 9, 5107-5118 (2016)

17. Zhao, Q.L., Li, X.Y.: A Bargmann system and the involutive solutions associated with a new 4-order lattice hierarchy. Anal. Math. Phys. 6, 237-254 (2016) 
18. Novikov, S.P., Manakov, S.V., Pitaevskii, L.P., Zakharov, V.E.: Theory of Solitons: The Inverse Scattering Method. Springer, Berlin (1984)

19. Flaschka, H.: On the Toda lattice. II: inverse-scattering solutions. Prog. Theor. Phys. 51, 703-716 (1974)

20. Hirota, R.: Exact N-soliton solution of the wave equation of long waves in shallow and nonlinear lattices. J. Math. Phys. $14,810-814(1973)$

21. Chen, H.H., Liu, C.S.: Bäcklund transformation solutions of the Toda lattice equation. J. Math. Phys. 16, 1428-1430 (1975)

22. Miura, M.R.: Backlund Transformation. Springer, Berlin (1978)

23. Hirota, R.: Nonlinear partial difference equations. IV. Bäcklund transformation for the discrete-time Toda equation. J. Phys. Soc. Jpn. 45, 321-332 (1978)

24. Mikhailov, A.V.: The reduction problem and the inverse scattering method. Physica D 1(2), 73-117 (1981)

25. Matveev, V.B., Salle, M.A.: Darboux Transformations and Solitons. Springer, Berlin (1991)

26. Sakhnovich, A.L.: Dressing procedure for solutions of nonlinear equations and the method of operator identities. Inverse Probl. 10, 699-710 (1994)

27. Cieśliński, J.L.: Algebraic construction of the Darboux matrix revisited. J. Phys. A 42, 404003 (2009)

28. Gu, C.H.: Soliton Theory and Its Applications. Springer, Berlin (1995)

29. Gu, C.H., Hu, H.S., Zhou, Z.X.: Darboux Transformations in Integrable Systems. Springer, Berlin (2005)

30. Park, Q.H., Shin, H.J.: Darboux transformation and Crum's formula for multi-component integrable equations. Physica D 157, 1-15 (2001)

31. Konstantinou-Rizos, S., Mikhailov, A.V., Xenitidis, P.: Reduction groups and related integrable difference systems of nonlinear Schrödinger type. J. Math. Phys. 56, 082701 (2015)

32. Yang, H.X.: Soliton solutions by Darboux transformation for a Hamiltonian lattice system. Phys. Lett. A 373, 741-748 (2009)

33. Zhang, X.Q., Yang, H.X., Zhao, J.C., Xu, X.X.: Soliton solution of the Toda lattice equation by the Darboux transformation. Chin. J. Phys. 44, 109-116 (2006)

34. Yu, F.J., Feng, S.: Explicit solution and Darboux transformation for a new discrete integrable soliton hierarchy with $4 \times 4$ Lax pairs. Math. Methods Appl. Sci. 40, 5515-5525 (2017)

35. Wen, X.Y: N-Fold Darboux transformation and soliton solutions for Toda lattice equation. Rep. Math. Phys. 68 $211-223(2011)$

36. Wen, X.Y., Hu, X.Y.: N-Fold Darboux transformation and solitonic interactions for a Volterra lattice system. Adv. Differ Equ. 2014, 213 (2014)

37. Wen, X.Y.: Elastic interaction and conservation laws for the nonlinear self-dual network equation in electric circuit. J. Phys. Soc. Jpn. 81, 114006 (2012)

38. Wen, X.Y., Yan, Z.Y., Malomed, B.A.: Higher-order vector discrete rogue-wave states in the coupled Ablowitz-Ladik equations: exact solutions and stability. Chaos 26, 123110 (2016)

39. Wen, X.Y., Wang, D.S.: Modulational instability and higher order-rogue wave solutions for the generalized discrete Hirota equation. Wave Motion 79, 84-93 (2018)

40. Xu, X.X: A deformed reduced semi-discrete Kaup-Newell equation: the related integrable family and Darboux transformation. Appl. Math. Comput. 251, 275-283 (2015)

41. Zhao, Q.L., Li, X.Y., Liu, F.S.: Two integrable lattice hierarchies and their respective Darboux transformations. Appl. Math. Comput. 219, 5693-5705 (2013)

42. Tu, G.Z.: A trace identity and its applications to theory of discrete integrable systems. J. Phys. A 23, 3903-3922 (1990)

43. Zhang, D.J., Chen, D.Y.: The conservation laws of some discrete soliton systems. Chaos Solitons Fractals 14, 573-579 (2002)

44. Toda, M.: Wave propagation in anharmonic lattices. J. Phys. Soc. Jpn. 23, 501-506 (1968)

45. Flaschka, H.: The Toda lattice. II. Existence of integrals. Phys. Rev. B 9, 1924-1925 (1974)

46. Gesztesy, F., Renger, W.: New classes of Toda soliton solutions. Commun. Math. Phys. 184, $27-50$ (1997)

47. Schiebold, C.: An operator theoretic approach to the Toda lattice equation. Physica D 122, 37-61 (1998)

48. Narita, K.: Solutions for the Mikhailov-Shabat-Yamilov difference-differential equations and generalized solutions for the Volterra and the Toda lattice equations. Prog. Theor. Phys. 99, 337-348 (1998)

49. Kodama, Y., Matsutani, S., Previato, E.: Quasi-periodic and periodic solutions of the Toda lattice via the hyperelliptic sigma function. Ann. Inst. Fourier 63, 655-688 (2013)

50. Nimmo, J.J.C.: Soliton solution of three differential-difference equations in Wronskian form. Phys. Lett. A 99, 281-286 (1983)

51. Takeno, S., Kisoda, K., Homma, S.: Approximate soliton solutions around an exact soliton solution of the Toda lattice equation. Phys. Lett. A 130, 279-282 (1988)

52. Ma, W.X., Maruno, K.: Complexiton solutions of the Toda lattice equation. Physica A 343, 219-237 (2004)

53. Ma, W.X., You, Y.C.: Rational solutions of the Toda lattice equation in Casoratian form. Chaos Solitons Fractals 22 395-406 (2004)

54. Hon, Y.C., Fan, E.G., Qin, Z.Y.: A kind of explicit quasi-periodic solution and its limit for the Toda lattice equation. Mod. Phys. Lett. B 22, 547-553 (2008)

55. Wazwaz, A.M.: Abundant solitons solutions for several forms of the fifth-order KdV equation by using the tanh method. Appl. Math. Comput. 182, 283-300 (2006)

56. Yang, J.K.: Nonlinear Waves in Integrable and Nonintegrable Systems. SIAM, Philadelphia (2010)

57. Trefethen, L.N.: Spectral Methods in MATLAB. SIAM, Philadelphia (2000) 